DIVISION OF THE HUMANITIES AND SOCIAL SCIENCES

CALIFORNIA INSTITUTE OF TECHNOLOGY

PASADENA, CALIFORNIA 91125

THE DYNAMICS OF DISTRIBUTIVE POLITICS

Marco Battaglini

Priinceton University

Thomas R. Palfrey

California Institute of Technology

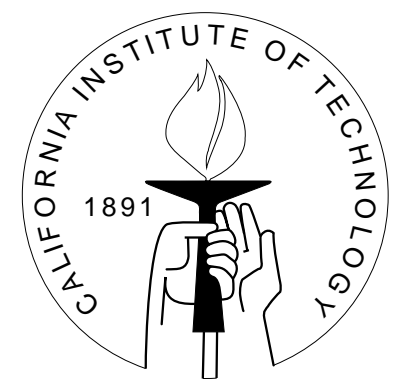

SOCIAL SCIENCE WORKING PAPER 1273 


\title{
The Dynamics of Distributive Politics ${ }^{1}$
}

\author{
Marco Battaglini ${ }^{2}$ \\ Thomas R. Palfrey ${ }^{3}$
}

June 2006 (Current Version July 27, 2007)

\footnotetext{
${ }^{1}$ We are grateful to the Center for Economic Policy Studies at Princeton University, the Princeton Laboratory for Experimental Social Science, and the National Science Foundation (SES-0450712, SES-0418150, and SES0617820) for financial support. Marco Battaglini gratefully acknowledges financial support from a NSF CAREER Award (SES-0547748), and the hospitality of the Kellogg School's MEDS department for the academic year 20062007. We thank Anna Bassi, Kyle Mattes, and Stephanie Wang for research assistance.

${ }^{2}$ Department of Economics, Princeton University, Princeton, NJ 08544. mbattagl@princeton.edu

${ }^{3}$ Division of the Humanities and Social Sciences, California Institute of Technology, Pasadena, CA 91125. trp@hss.caltech.edu
} 


\begin{abstract}
We study dynamic committee bargaining over an infinite horizon with discounting. In each period a committee proposal is generated by a random recognition rule, the committee chooses between the proposal and a status quo by majority rule, and the voting outcome in period $t$ becomes the status quo in period $t+1$. We study symmetric Markov equilibria of the resulting game and conduct an experiment to test hypotheses generated by the theory for pure distributional (divide-the-dollar) environments. In particular, we investigate the effects of concavity in the utility functions, the existence of a Condorcet winning alternative, and the discount factor (committee "impatience"). We report several new findings. Voting behavior is selfish and myopic. Status quo outcomes have great inertia. There are strong treatment effects, that are in the direction predicted by the Markov equilibrium. We find significant evidence of concave utility functions.
\end{abstract}

Keywords: Dynamic bargaining, voting, experiments, divide-the-dollar, committees.

JEL Classification: D71, D72, C78, C92. 


\section{Introduction}

The large redistributive programs that have characterized western democracies since the end of World War II -pension, health, and disability plans, for example- share a common characteristic. Once one of these programs is approved by congress, it remains in force until explicitly revised. This feature makes the politics of redistribution an intrinsically dynamic game that can not be studied as a simple static struggle for resources among different constituencies, or even as a sequence of independent struggles. A policy chosen today will be the status quo tomorrow. In choosing the optimal policy, a policy maker should not only consider the direct effect of the policy today, but also the indirect effect that the policy has for future policy decisions. In the short run the policy maker may propend toward a policy that favors only his constituency; when the long run is considered, however, a more moderate policy may be preferred: a moderate status quo favorable to a larger constituency will be harder to overturn in the future.

Recent theoretical work has put particular emphasis on the dynamic nature of redistribution producing a rich assortment of predictions. ${ }^{1}$ This literature raises three natural questions: To what extent do game theoretic models accurately predict behavior in a dynamic policy game? Can the models be improved to better explain empirical evidence? If so, how?

In this paper we make a step in answering these questions by studying equilibrium behavior in a simple dynamic model of committee bargaining with endogenous status quo and by presenting the first laboratory experiment on this class of games. ${ }^{2}$ We consider an infinite horizon model in which a committee of three agents has to divide a dollar at every period. At the beginning of a period a member of the committee is selected by a random rule to propose a division to the committee. The committee then chooses by majority rule between the proposal and a given status quo. The selected policy is implemented and it becomes the new status quo. With a positive probability the game is repeated exactly as before, but with the new status quo; with the complementary probability the game is terminated. The policy choice in period $t$, therefore will affect the bargaining game at $t+1$, and indirectly in the following period as well.

\footnotetext{
${ }^{1}$ Among the most recent works, see Azzimonti [2006], Barron and Herron [2003], Battaglini and Coate [2006, 2007a,2007b], Diermeier and Fong [2007], Duggan and Kalandrakis [2007], Kalandrakis [2006], Penn [2007].

${ }^{2}$ Previous important experimental work on legislative bargaining games is provided by McKelvey [1991] and, more recently, by Diermeier and Morton [2004] Diermeier and Gailmard [2006] and Frechette, Kagel and Morelli [2003, 2005a,b,c and 2007]. All this work, however, focuses on static environments inspired by the seminal paper by Baron and Ferejohn [1989] in which a given amount of resources is allocated only once.
} 
We study this model because similar models have been theoretically studied by a number of authors (Epple and Riordan [1987], Baron [1996], Baron and Herron [2003], Kalandrakis [2004] and [2006] and Duggan and Kalandrakis [2007]) and it is therefore a natural starting point. Despite its simplicity, a complete understanding of behavior in this game has proven so far elusive. When agents are risk neutral, Kalandrakis [2004] has shown that this game has a Symmetric Markov Equilibrium (SME) in which committee members behave myopically, maximizing their current utility. In this equilibrium, therefore, proposers succeed in appropriating all or almost all the dollar in every period as if the game were a sequence of one period games. Baron [1987] and Baron and Herron [2003] however have argued that with more general utilities, agents have stronger incentives for dynamic strategic behavior. Concavity in the utility function leads them to more equitable outcomes. Since, however, equilibrium behavior in a legislative bargaining game with general utilities has not been characterized yet, the importance of this phenomenon is not known in general.

To investigate these issues and provide a theoretical benchmark for the experimental evidence, we first study SME in complex redistributive bargaining environments with concave utilities by numerical methods. We show that Baron's conjecture is correct by computing an equilibrium in which as concavity increases, equilibrium outcomes become more equitable. ${ }^{3}$ We also solve a simplified version of the dynamic bargaining game with a restricted set of possible division of the pie. This has two advantages. First, it allows us to show the existence of and fully characterize a SME which possesses the same features of the more general case with a continuum of states, but also to prove its uniqueness, an important properties for comparative statics and the interpretation of experimental evidence. Second, it allows us to analyze bargaining in policy spaces with a Condorcet Winner (which never exists when the policy space is too rich as in the standard divide the dollar game). One of the central principles of static models of committee decision making is that Condorcet winners will prevail. In the environment that we study, even with a Condorcet winner we predict the stability of dynamic regimes where non-Concorcet winners prevail indefinitely.

These theoretical findings provide a rich set of predictions that we can test in the laboratory. We consider an experimental design that varies the environment across three dimensions. One dimension is whether the environment is a (nearly) continuous divide the dollar setting, versus a more constrained set

\footnotetext{
${ }^{3}$ In independent work, Duggan and Kalandrakis [2007] have recently also shown a similar phenomenon by numerical methods (though with a different numerical algorithm). They, however, have limited the numerical analysis to a simpler game with two players and unidimensional policy space.
} 
of allocations. The second dimension, applied to the finite environment, is the effect of the existence of a Condorcet winner. The third dimension, applied to the continuous environment, is the effect of long run incentives, which we study by varying the discount factor of the committee members - comparing "patient" legislatures or committees with "impatient" ones.

Our experimental findings allow a clear evaluation of the ability of theoretical models to predict empirical behavior. In environments where bargaining is over a limited set of states, the "standard" theoretical model assumed in the literature (in which utilities are linear and agents play according to Nash equilibrium) is consistent with many features of the data, but with some exceptions which we discuss. The model predicts, in particular, the difference in behavior that we observe between the case in which there is a Condorcet winner among the alternatives or not. When bargaining is over more complicated state spaces (as in the unit simplex), however, the standard model performs less well. The model predicts highly unequal outcomes in which in each period one agent appropriates most of the resources: however, we rarely observe such outcomes, on the contrary, we observe a significant frequency of allocations in which resources are evenly distributed among all participants. We can however show that this type of behavior is not necessarily evidence of social preferences or non strategic behavior. Indeed, there is little or no evidence indicating any preferences for fairness. Rather, our main experimental findings are consistent with selfish preferences and concave rather than linear utilities, and we fit such a model to the data. Players tend to make proposals that maximize their payoff at the expense of others when it is optimal to do so (as when they are favored by the status quo); and voting behavior is overwhelmingly myopic and selfish in all treatments.

The rest of the paper is organized as follows. The next section lays out the model. Section 3 characterizes the theoretical properties of the model. Section 4 describes the experimental design. Section 5 describes the findings of the experiment. We conclude in section 6.

\section{Model}

We consider the problem faced by a set of $N$ agents who repeatedly bargain over a set of outcomes $X$. In each period $t=1,2,3 \ldots$ a policy $x_{t}$ is chosen by the agents. The bargaining protocol with which a the policy $x_{t}$ is chosen is as follows. At the beginning of each period an agent is chosen by nature to propose an outcome $y_{t}$. The floor votes on this outcome following a $q$ rule, where $q \in[1, N]$. If the number voting in favor is greater than or equal to $q$, the proposal is accepted, and $y_{t}$ chosen at $t$. If the proposal 
is voted by less than $q$ agents, a status quo policy $s_{t}$ is implemented. The status quo $x_{0}$ is exogenous at $t=1$, and assumed to coincide with the outcome implemented in the previous period for $t>1$, that is $s_{t}=x_{t-1}$. Each agent can be recognized as a policy proposer: the probability that agent $i$ is recognized as proposer is $\frac{1}{3}$, so the probability of being recognized is history invariant. This bargaining protocol was introduced by Epple and Riordan [1987] and then studied by Baron [1996], Baron and Herron [2003], Kalandrakis [2004].

Agents have a Von Neuman Morgenstern per period Utility $U_{i}: X \rightarrow \mathbb{R}$, which is assumed to be continuous and (weakly) quasi concave. The policy implemented in period $t, x_{t}$, therefore induces an n-tuple of utilities $\left(U_{i}\left(x_{t}\right)\right)_{i=1}^{N}$. The utility of an infinite sequence of policies, $x=\left\{x_{1}, . ., x_{t}, \ldots\right\}$ is given by $U_{i}^{\delta}(x)=(1-\delta) \sum_{t=1}^{\infty} \delta^{t-1} U_{i}\left(x_{t}\right)$ where the discount factor, $\delta$ is assumed to be strictly less than 1 .

Many examples of this general framework can be constructed. We will focus on two:

Example 1 (Divide the Dollar). The agents have to divide a pie of size $K$. An allocation is vector $\left(x_{t}^{i}\right)_{i=1}^{N}$ where $x^{i} \geq 0$ and $\sum_{i=1}^{N} x^{i}=K$. Each agent is interested only in the size of the pie that he receives.

Example 2 (Public Goods). In this case $X$ is a collection of projects $\left\{x_{1}, \ldots, x_{j}\right\}$, where each project is associated with a utility impact on the agents: $\left(U_{i}\left(x_{k}\right)\right)_{i=1}^{N}$ for any $x_{k} \in X$.

An outcome in period $t$ is given by the initial status quo, Nature's choice of a proposer $\iota_{t} \in\{1, \ldots, N\}$, the proposed policy $y_{t} \in X$, the vector of votes $\eta_{t} \in\{1,0\}^{N}$. Let $h_{t}=\left\{\iota_{t}, y_{t}, \eta_{t}, x_{t}\right\}$ be an outcome at time $t \geq 1$ where $x_{0}$ is exogenously given and $x_{t}=\chi\left(h_{t}\right)$ is the policy choice implied by the voting rule and the history $h_{t}$. An history $h^{t}$ is defined as $h^{t}=\left\{h_{1}, \ldots . h_{t}\right\}$; the set of histories at time $t$ is $H^{t}$. A strategy for agent $i$ is a set of functions $s_{i}(t)=\left(\rho_{i}(t), \sigma_{i}(t)\right)_{t=1}^{\infty}$ : where $\rho_{i}(t): H^{t-1} \rightarrow \Delta X$ describes the proposal choice $(\Delta X$ is the set of randomizations over $X)$; and $\sigma_{i}(t): H^{t-1} \times\{1, . ., N\} \times X \rightarrow[0,1]$ associates to each history $h^{t-1}$, proposer choice and proposal in $X$ a probability to vote to accept (1) or reject $(0)$.

In a sequential equilibrium the strategies are measurable with respect to the entire history set $H^{t}$. A Markov strategy, on the contrary, is measurable only with respect to the status quo $x_{t}$ and the events that occur in period $t: \rho_{i}: X \rightarrow \Delta X, \sigma_{i}: X \times\{1, . ., N\} \times X \rightarrow[0,1]$. A Markov Equilibrium is a Nash equilibrium in Markov Strategies. In the following analysis we will focus on Markov equilibria. To each Markov equilibrium and each agent $i$, we can associate a function $v_{i}(\theta)$ which represents the expected 
continuation value of agent $i$ when the status quo (current policy) is $\theta$ before he knows who will be the proposer. Given this we can define the function $u_{i}(\theta)=U_{i}(\theta)+\delta v_{i}(\theta)$ as the expected utility of agent $i$ if policy $\theta$ is implemented in a representative period; and the function $u_{i}\left(\theta^{\prime} ; \theta\right)$ as the expected utility of agent $i$ if he proposes $\theta^{\prime}$ when the status quo is $\theta$.

When the agents are identical it is natural to consider equilibria in which agents behave and treat the other agents in the same way. An equilibrium is anonymous if for any $\theta, x, y: u_{i}(x ; \theta)=u_{i}(y ; \theta)$ implies $\rho_{i}(x \mid \theta)=\rho_{i}(y \mid \theta)$, and $u_{i}(x)=u_{i}(\theta)$ implies $\sigma_{i}(x \mid \theta)=\frac{1}{2}$ (where $\rho_{i}(x \mid \theta)$ is the probability that $x$ is proposed by $i$ in state $\theta$, and $\sigma_{i}(x \mid \theta)$ is the probability that voter $i$ votes for $x$ when the status quo is $\theta$ ). Intuitively an agent cares only about his expected payoff, not about the particular states that achieves the payoffs. An equilibrium is symmetric if $s_{i}(t)=s_{j}(t)$ for any $i, j$ and strategies are anonymous. A Markov equilibrium is in stage undominated strategies if in each stage no agent uses a strategy that is weakly dominated given his equilibrium value function $v_{i}(\theta)$. From now on we will focus on symmetric anonymous Markov equilibria in stage undominated strategies, and we refer to them simply as equilibria.

\section{Theoretical predictions}

In this section we describe the equilibrium properties of the game described in Section 2 under additional assumptions on the policy space. We focus exclusively on the case of $N=3$ and $q=2$ In Section 3.1 we consider equilibrium behavior in a standard divide the dollar game. In Section 3.2 we discuss the case in which the policy space is restricted to a more limited set of alternatives.

\section{III.1 The divide the dollar game}

Despite its simplicity, there is no known characterization of the divide the dollar bargaining game described above for general utility functions. ${ }^{4}$ To obtain predictions that we can test using our experimental data, we turned to numerical methods. Here we describe properties of the numerically computed equilibrium under the parameter specifications used in the experiments: three agents, a pie of size 60 and discount a factor equal to either 0.83 or 0.75 .

\footnotetext{
${ }^{4} \mathrm{~A}$ characterization of an equilibrium in the divide the dollar game is available only for the case of linear utilities (see Kalandrakis [2003]). We will discuss this equilibrium below.
} 


\section{III.1.1 Numerical Computation of the Markov Equilibrium}

We compute a Markov equilibrium for the family of utility functions with constant relative risk aversion:

$$
U_{i}\left(x_{i} ; \gamma\right)=\frac{1}{1-\gamma}\left(x_{i}\right)^{1-\gamma} \quad \forall i=1,2,3
$$

where $x_{i}$ is the share received by agent $i$. The coefficient of relative risk aversion $\gamma$ measures the curvature of the utility function: the higher is $\gamma$, the more concave is utility. For simplicity, in this section we discuss two polar cases: the linear case, $\gamma=0$, and a strictly concave case, $\gamma=0.95 .^{5}$

Equilibria were computed as the limit of Markov Logit Quantal Response Equilibria (MLE) by gradually reducing noise in the agents reaction functions. This smooths out the best response correspondence, which is helpful in the numerical computation. In the logit version of quantal response equilibrium, as defined for extensive form games, each player, at each information set uses a behavioral strategy where the log probability of choosing each available action is proportional to its continuation payoff, where the proportionality factor, $\lambda$, can interpreted as a responsiveness (or rationality) parameter. The continuation payoffs are computed using the MLE strategies of all future play in the game, as, for example, in the definition of continuation payoffs in a sequential equilibrium (Kreps and Wilson 1982). ${ }^{6}$ Markov perfect equilibria can be found as limits of MLE because for very high values of $\lambda$ players choose best responses with probability approaching 1 , so limit points of the MLE correspondence, as $\lambda \rightarrow \infty$ are Markov equilibrium. Moreover, in generic games there is unique Markov perfect equilibrium that is selected as the limit of the unique connected path in the equilibrium graph that has a solution for every value of $\lambda \geq 0$.

We solved the game using discrete approximation of a unit simplex where allocations are in increments of 5 . This reduces the set of states to 91 . Formally, the policy space is:

$$
X:=\left\{x=\left(x_{1}, x_{2}, x_{3}\right) \text { s.t. } \forall i \exists t \in \mathbb{N}, x_{i}=5 t, \quad \sum_{i=1}^{3} x_{i}=60\right\} .
$$

Given the smooth properties of this MLE path, there is a simple and (relatively) fast path-following algorithm which will find this solution. It is simple because we know the solution at $\lambda=0$ : all (behavioral) strategies are chosen with equal probability, and this implies the unique value function. Hence, we begin with the solution at $\lambda=0$ and can use that solution as the starting value to find the MLE for an incrementally larger value, say $\lambda=\epsilon$. Because we are guaranteed that for small enough $\epsilon$ the starting value obtained from $\lambda=0$ is very close the the solution at $\lambda=\epsilon$, the fixed point algorithm will find a

\footnotetext{
${ }^{5}$ In later sections we will use experimental data to obtain a maximum likelihood estimate of $\gamma$.

${ }^{6} \mathrm{~A}$ precise definition of this equilibrium concept is presented in Appendix 1.
} 
solution at $\epsilon$ very quickly. Then, we use the solution at $\lambda=\epsilon$ to compute the solution at $\lambda=2 \epsilon$ and so forth, thereby tracing out the unique MLE path that converges to a Markov equilibrium of the game. There are some numerically tricky issues when $\lambda$ becomes very large, and the algorithm takes several hours but conceptually it is quite simple, and convergence is not difficult to achieve.

\section{III.1.2 Steady State Equilibrium Dynamics}

The agent proposal strategy associates to each status quo a probability of proposing a state. The voting strategies associate a probability of voting yes to each possible status quo - proposal pair. Because the equilibrium strategy space is so large, to describe the properties of equilibrium behavior it is convenient to use the stationary distribution over outcomes induced by equilibrium strategies. The equilibrium strategies generate a Markov process with a stationary transition matrix. This transition matrix associates

each state $x^{\prime} \in X$ to a probability distribution $\varphi\left(x \mid x^{\prime}\right)$ over states $x \in X$ in the following periods. For a given initial distribution $P^{0}(x)$ over the status quos, we can therefore define the equilibrium distribution of states at $t$ recursively as:

$$
P^{t}(x)=\sum_{x^{\prime} \in X} \varphi\left(x \mid x^{\prime}\right) P^{t-1}\left(x^{\prime}\right) .
$$

The probability function $P^{t}(x)$ converges to a stationary distribution $P^{*}(x)$ as $t \rightarrow \infty$. This distribution represents the frequency of the states that we would expect to observe in the long run, so it provides one of the fundamental properties of the Markov equilibrium.

For descriptive purposes, we cluster the states in coarser regions. Figure 1 describes a partition of the states in 7 regions. The $D$ regions correspond to dictatorial allocations where one player receives the lion's share of the pie. The $M$ regions correspond to majoritarian allocations where a coalition of two players receive most of the pie, with nearly equal shares, while the third player receives only a small amount or nothing. The $U$ region consists of universal allocations, where the pie is equally, or nearly equally, shared. Conditional on being, say, in $D 1$ we can use the stationary distribution of the computed Markov equilibrium to derive the probability of transition to state $M 12$. Doing this for all pairs of regions gives a representation of the steady state equilibrium dynamics of the infinitely repeated game in a simple $7 \times 7$ matrix. This allows us to describe the dynamics in a compact way.

Linear utilities. We start with the discussion of the equilibrium with linear utilities and $\delta=0.83$. In choosing how to allocate the pie, a proposer is faced with a trade off between short run and long 


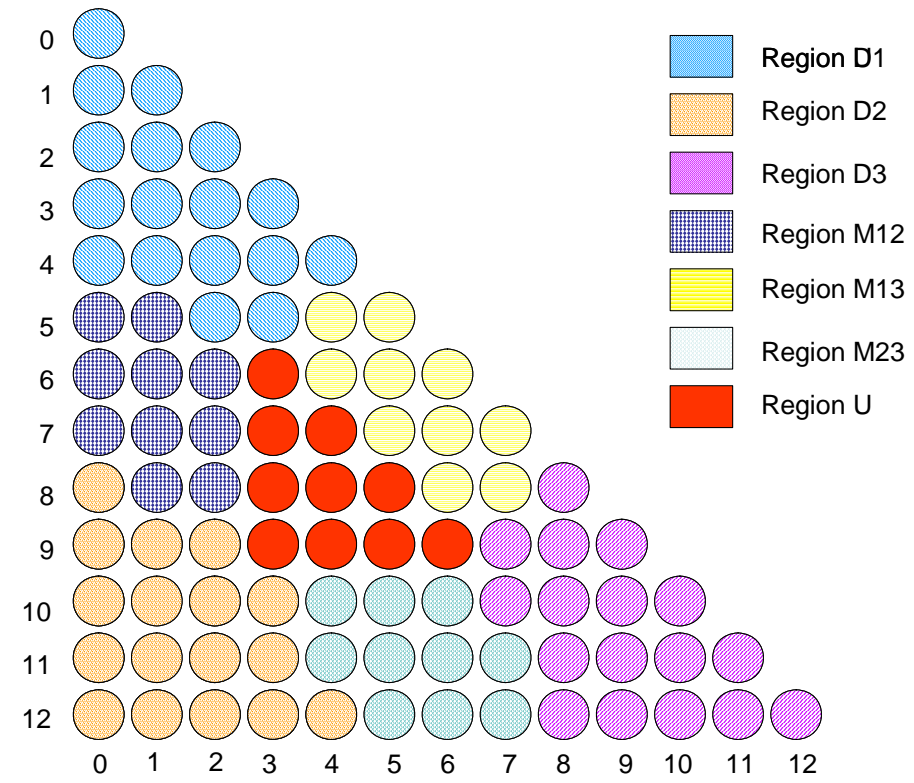

Figure 1: Allocation regions. The vertical coordinate represents Agent 1's payoff, the horizontal represents Agent 3's payoff. The payoff of Agent 2 is the residual.

run effects of the allocation. In the short run, a proposer is facing a simple problem: if the proposer were completely myopic $(\delta=0)$, he would attempt to form a minimal winning coalition and maximize his immediate payoff. In the long run, however, the game is more complicated because a state that maximizes his payoff today may reduce his payoff in the future.

To see which effect dominates when agents are risk neutral, consider the equilibrium transition matrix. Table 2 represents the transition matrix using the states described in Figure 1. Given the symmetry of the equilibrium we have only 3 regions to consider: if we are in D1, in M12 or in U: the remaining cases will be the same. The dynamics implied by (2) is therefore easily represented in Figure 3.A which describes the transition probabilities from these three states.

Figure 3.A makes clear that the short run effect dominates. Assume the initial state is in D1, where agent 1 receives most of the pie. In this case the state will stay at D1 with $34 \%$ probability and move to Dj j=2,3 with $33 \%$ probability: so with $100 \%$ probability the state will remain in the extreme regions. This occurs because in D1 each agent will propose almost all the payoff for himself, with a minimal share going to a single coalition partner. The probability that we remain in D1 is higher because some of these proposals can be rejected with positive probability.

It is interesting to note the dynamics from a status quo in $\mathrm{U}$. In this case the state does not jump 


\begin{tabular}{|c|c|c|c|c|c|c|c|c|}
\hline \multirow[t]{2}{*}{$\delta=.83$} & & \multicolumn{7}{|c|}{ Status Quo (t+1). $\delta=.83 q=0$} \\
\hline & & D1 & D2 & D3 & M12 & M13 & M23 & U \\
\hline Status & D1 & .34 & .33 & .33 & 0 & 0 & 0 & 0 \\
\hline \multirow[t]{6}{*}{ Quo (t) } & D2 & .33 & .34 & .33 & 0 & 0 & 0 & 0 \\
\hline & D3 & .33 & .33 & .34 & 0 & 0 & 0 & 0 \\
\hline & M12 & .33 & .33 & .25 & .08 & 0 & 0 & 0 \\
\hline & M13 & .33 & .25 & .33 & 0 & .08 & 0 & 0 \\
\hline & M23 & .25 & .33 & .33 & 0 & 0 & .08 & 0 \\
\hline & U & .001 & 0.01 & 0.01 & .31 & .31 & .31 & .03 \\
\hline
\end{tabular}

Figure 2: Transition probabilities in the seven region partition with linear utilities.

directly to a region $D i \quad i=1,2,3$ with high probability (in total only $3 \%$ of the time). More likely the state will transit to a state Mij $i, j=1,2,3$ (over $90 \%$ of the time). This because it is very difficult for $i$ to convince any other agent to vote for a $D i$ proposal. This can only happen if the state in $\mathrm{U}$ is bordering a region $D j, j \neq i$, by offering to $k \notin\{i, j\}$ (a currently disadvantaged agent) a more advantageous payoff in $\mathrm{Di}$. From a state $\mathrm{Mij}$, however, the system moves to a D state with high probability, over $90 \%$ of the time.

In the long run, therefore we would expect the state to rotate around regions $D 1, D 2$ and $D 3$. This myopic behavior can be clearly seen in the stationary distribution of outcomes represented in Figure 4.A. In the long run most of the mass of the distribution of states is on the extremes: that is on states in which a single agent receives a payoff between 50 and 60 . When agents are risk neutral, therefore, they behave as if they were myopic choosing allocations that maximize their current payoff.

This finding is consistent with the analysis in Kalandrakis [2004] who characterized an equilibrium of the bargaining game when the state space is the unit simplex (and so the unit of account is infinitesimal). There is, however, a significant difference. Kalandrakis [2004] shows that in the long run only the most extreme states are chosen (i.e., only states in which one agent receives 60 ). In the equilibrium presented above, this does not occur: indeed with a significant probability at least one of the other agents receives a positive payment. This difference is due to the fact that in the model studied here the proposer must divide the pie in units of $1 / 12$ of the total size and because we are studying a neutral equilibrium. The proposer does not want to make offers that leave the other players just indifferent, because in this case neutrality implies they would vote in favor of the proposal only $1 / 2$ of the time. The proposer, however, can not induce strict preferences by offering an infinitesimal increase in payment. He is therefore forced 

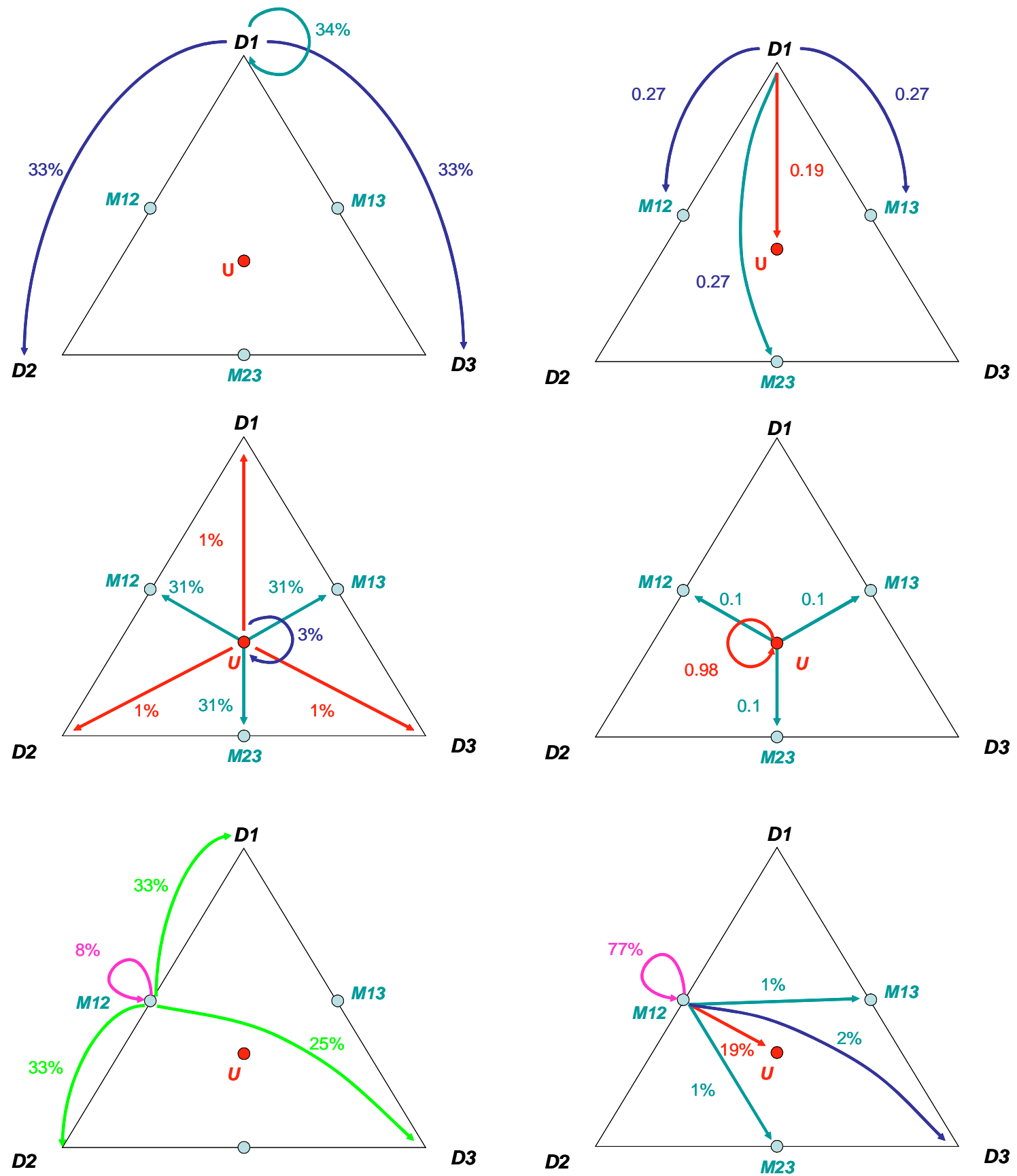

Case A: gamma=0

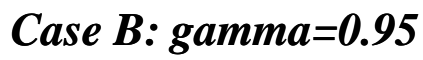

Figure 3: Theoretical transition probabilities when $\delta=0.83$. The left graphs are for the linear utility equilibrium and the right graphs are for the concave utility $(\gamma=.95)$ equilibrium. The top graphs illustrate transitions from D regions; the middle graphs from U; the bottom graphs from $\mathrm{M}$. 


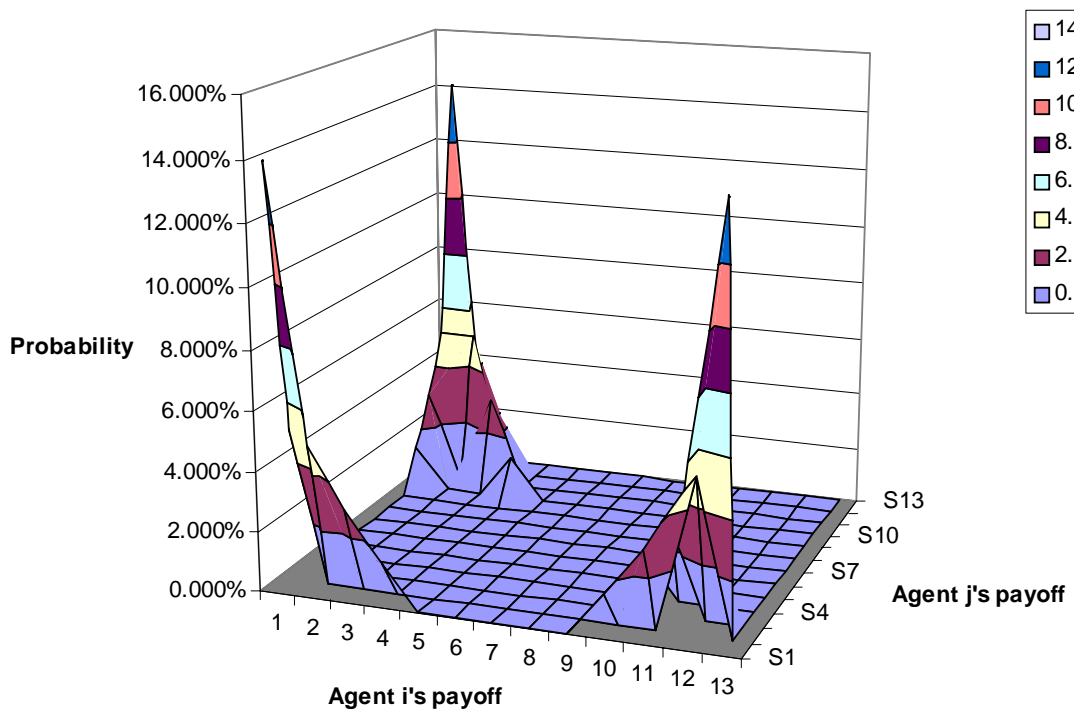

Case A: gamma $=0$

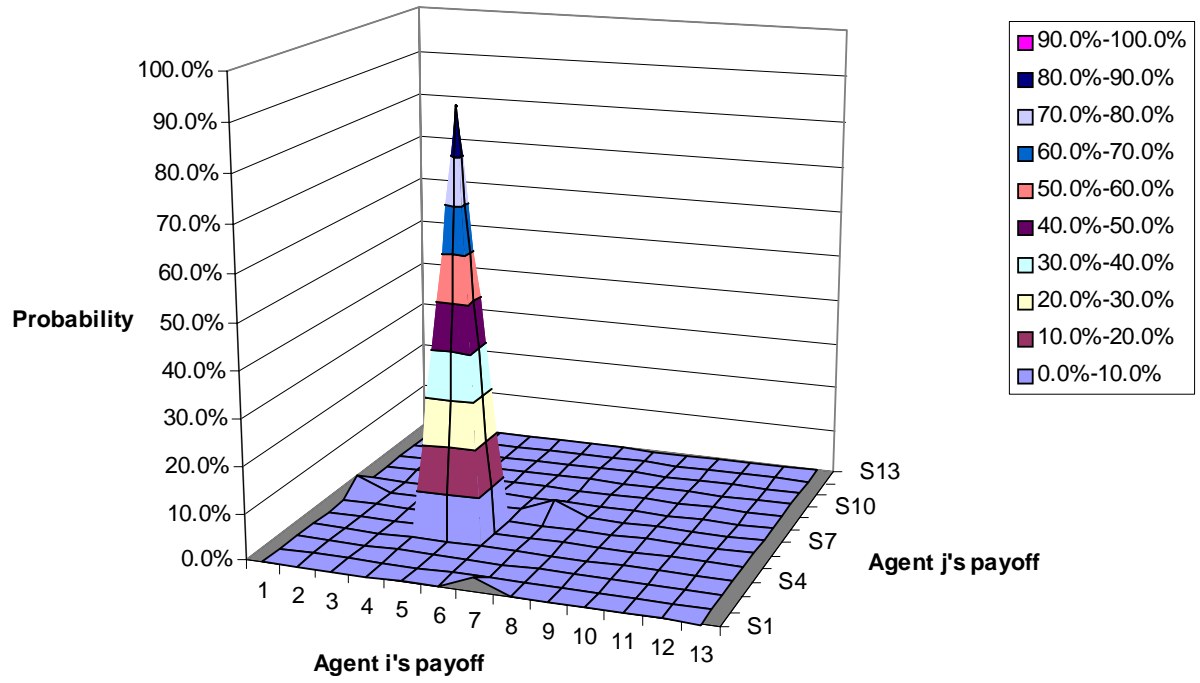

Case B: gamma $=0.95$

Figure 4: Stationary distribution of allocations $(\delta=.83)$. The top graph is for $\gamma=0$ and the bottom graph is for $\gamma=.95$. 


\begin{tabular}{|c|c|c|c|c|c|c|c|c|}
\hline \multicolumn{1}{|c|}{ Theoretical Transition Matrix of the 7 regions: $q=0.95, \delta=0.83$} \\
\hline \multicolumn{10}{|c|}{$Q_{t+1}$} \\
\hline$S Q_{t}$ & D1 & 0 & 0 & 0 & 0.27 & 0.27 & 0.27 & 0 \\
\hline & D2 & 0 & 0 & 0 & 0.27 & 0.27 & 0.27 & 0 \\
\hline & D3 & 0 & 0 & 0 & 0.27 & 0.27 & 0.27 & 0 \\
\hline & M12 & 0 & 0 & 0.2 & 0.77 & 0.01 & 0.01 & 0.19 \\
\hline & M13 & 0 & 0.2 & 0 & 0.01 & 0.77 & 0.01 & 0.19 \\
\hline & M23 & 0.2 & 0 & 0 & 0.01 & 0.01 & 0.77 & 0.19 \\
\hline & U & 0 & 0 & 0 & .01 & .01 & .01 & 0.98 \\
\hline
\end{tabular}

Figure 5: Transition probabilities in the 7 region partition with linear utilities when $q=0.95$

to offer something to his coalition partner. ${ }^{7}$

Concave utilities With strictly concave utility functions, agents are averse to sequences of outcomes in which the status quo changes at every period. Hence the incentives for more symmetric distributions are greater because such distributions generate less variance across time. The least efficient outcome is one where the proposer appropriates the entire pie in each period. Though in this case an agent is receiving 20 on average (60 one-third of the time and 0 two-thirds of the time), this gives a much lower discounted utility than receiving exactly 20 in every period. Proposers can avoid such "rotating dictator" outcomes by choosing a division that is closer to the centroid of the simplex. By allocating a higher share to an agent, the proposer exposes himself less to expropriation in the future, because it makes it harder for a future proposer to extract a larger share of the surplus by forming a coalition with an excluded agent. A proposal close to the centroid is harder to overturn and reduces the volatility of the proposer's future payoffs.

To see that the myopic behavior is no longer optimal in equilibrium consider Table 5 , which describes the 7 region transition matrix in the case with $\gamma=0.95$, and Figure 3.B which represents the 3 leading cases. The differences between Figure 3.B and Figure 3.A $(\gamma=0)$ are striking. Starting from a state in region D1, when $\gamma=0.95$ we never move to a region D1, D2 or D3. Most of the time, we move to a state M12, M13 or M23 (over $80 \%$ of the time) and $19 \%$ of the time we move directly to region U. When the state is in a majoritarian region either we remain in the same region ( $77 \%$ of the time) or we again move to $\mathrm{U}$ with high probability, nearly $20 \%$ of the time. Once we reach region $\mathrm{U}$, however, it is very

\footnotetext{
${ }^{7}$ Kalandrakis [2004] assumes that, when indifferent, a voter votes for the proposal with probability one.
} 
difficult to exit: with $98 \%$ probability the state remains in U.

The long run incentives to move toward the center are clear in the stationary distribution represented in Figure 4.B. While with linear utilities the probability that agent $i$ receives a payoff between 20 and 40 is 0 , it is over $96 \%$ with $\gamma=0.95$.

The tendency of outcomes to cluster around the centroid confirms the phenomenon identified by David Baron (Baron [1996]) in a unidimensional case. To our knowledge, Figures 3.B and 4.B represents the first equilibrium construction of a stationary bargaining game with concave utility functions proving Baron's idea in a multidimensional world. ${ }^{8}$

\section{III.2 Simplified Divide-the-Dollar}

The analysis of the previous section has two features that lead us to examine a simplified divide-thedollar problem where there are only four possible splits. First, even under the restricted class of Markov equilibria we cannot rule out multiple equilibria. Second, because the policy space is an approximation of the unit simplex, it is an environment without Condorcet winners.

Hence we consider two cases. In both cases, the 3 -way equal split which we call the universal allocation, is feasible. The two cases then differ in the other three allocations. In the first case (CW, which stands for "Condorcet Winner"), the universal allocation is a Condorcet Winner: that is, it is myopically preferred by a majority of voters in any pairwise comparison with the other three allocations. The second case (NCW, which stands for "No Condorcet Winner") we consider includes three majoritarian allocations, where the pie is divided equally between two committee members, and the third committee member receives 0 .

\section{III.2.1 No Condorcet Winner (NCW)}

Consider a bargaining game with a set of players $N=\{1,2,3\}$ and four states $X=\left\{x_{0}, x_{1}, x_{2}, x_{3}\right\}$ that induce payoffs described by the following matrix $S$ :

$$
\left[\begin{array}{cccc} 
& 1 & 2 & 3 \\
x_{0} & 20 & 20 & 20 \\
x_{1} & 0 & 30 & 30 \\
x_{2} & 30 & 0 & 30 \\
x_{3} & 30 & 30 & 0
\end{array}\right]
$$

\footnotetext{
${ }^{8}$ Baron and Herron [1999] conjectured that the phenomenon would extend to multidimensional environment. They numerically computed the equilibrium for a game with finite horizon and shown that proposals tend to be biased to the centroid. Duggan and Kalandrakis [2007] show the phenomenon in a one dimensional environment with 2 players.
} 
where the rows describe the states, and the column the players: the matrix specifies the per period utility of an agent for each state. ${ }^{9}$ In this game there are only two possibilities. Either the outcome is egalitarian if state $x_{0}$ is chosen; or the outcome is strictly majoritarian: a minimal wining coalition of players share the dollar and leave the remaining player with nothing. There is no Condorcet winner.

The following result characterizes the symmetric equilibrium when payoffs are described by (1):

Proposition 1. When payoffs are as (1), with linear utilities there is a unique equilibrium. Each agent $i$ proposes following history independent strategy $\rho_{i}\left(x_{j} \mid x\right)=\rho_{i}\left(x_{k} \mid x\right)=\frac{1}{2} \forall j, k \neq\{i, 0\}$ and $\forall x$, and votes for the alternative that offers the highest immediate payoff, mixing with equal probability when indifferent. This remains an equilibrium if the agents have the same strictly increasing utility function.

This result provides clear-cut predictions that can be tested in the laboratory. The proposal behavior and the voting behavior does not depend on the initial status quo. Specifically, in every round, equilibrium proposal strategies are mixed, with equal probability on the two allocations that give the agent 30 . Voting behavior is myopic, with indifference leading to uniform mixing. This Markov equilibrium generates a unique transition probability function. From a status quo $x_{i}$ the state remains at $x_{i}$ with probability $\frac{2}{3}$ and moves to a state $x_{j}$ with probability $\frac{1}{3}$, and never moves to $x_{0}$. From status quo $x_{0}$, the state moves to each other state $x_{i}$ with probability $\frac{1}{3}$. Proposals of $(20,20,20)$, therefore, never occur in equilibrium, so votes involving $(20,20,20)$ can only occur in the very first round, and only if $(20,20,20)$ is the initial status quo.

It is interesting to compare Proposition 2 with the results in the case of a purely distributive policy space when $\gamma=0$, as implicitly assumed here. Even with a limited number of states, the model predicts that decision are taken by minimal winning coalitions. As in the case of a purely distributive policy space, here too the equilibrium does not select the symmetric allocation. The asymmetry of payoffs in equilibrium is not as marked as in the model presented in the previous section or in the continuous case, but the reason is that there is an exogenous limit to the ability to appropriate payoffs: the equilibrium outcomes are as asymmetric as possible. As in the case with a continuum of states, equilibrium behavior of agents mimics myopic behavior, behaving exactly as agents with a zero discount factor, even though all agents are in fact strategic and forward looking. Here, however, Proposition 2 makes clear that there is a unique symmetric equilibrium with linear utilities: so it provides a sounder base to test the theoretical prediction with laboratory experiments.

\footnotetext{
${ }^{9}$ In (1) we assume that the sum of payoffs is 60 because this is the size of the pie that we use in the experiments.
} 
We also observe that concavity $(\gamma>0)$ does not affect this equilibrium. That is, regardless of the concavity of the utility function, the equilibrium identified in Proposition 1 persists. To see this, note that $i$ 's equilibrium continuation value of the universal allocation is exactly the same as the continuation value from the $i$ state.

\section{III.2.2 Condorcet Winner (CW)}

With finite states there can be a Condorcet winner. Consider the bargaining game described by the following set of four allocations:

$$
\left[\begin{array}{cccc} 
& 1 & 2 & 3 \\
x_{0} & 20 & 20 & 20 \\
x_{1} & 30 & 15 & 15 \\
x_{2} & 15 & 30 & 15 \\
x_{3} & 15 & 15 & 30
\end{array}\right]
$$

As in (1) we have a symmetric policy $\left(x_{0}\right)$; now, however, in policies $x_{i} i=1,2,3$ one agent receives a payoff double to the payoff of the other players. The Condorcet winner is $x_{0}$.

Consider a strategy profile, in which voters vote "myopically" for the alternative that offers the highest per period payoff, mixing with equal probability when indifferent; and a proposer $i$ proposes his favorite alternative $\left(x_{i}\right)$. We call a strategy profile with these characteristics a myopic strategy.

A myopic strategy profile defines a well defined value function. Assume that agents have linear utilities. The continuation value in state $x_{0}$ is the simplest to find, since the outcome transits out of this state with zero probability, so

$$
v_{i}\left(x_{0}\right)=\frac{20}{(1-\delta)}
$$

for any $i$. Given this, the remaining value functions can also be easily found by backward induction. When the state is $x_{i}$, the value function $v_{i}\left(x_{i}\right)$ of agent $i$ is

$$
\begin{aligned}
v_{i}\left(x_{i}\right) & =\frac{1}{3}\left(30+\delta v_{i}\left(x_{i}\right)\right)+\frac{2}{3}\left[\frac{1}{2}\left(30+\delta v_{i}\left(x_{i}\right)\right)+\frac{1}{2}\left(15+\delta v_{i}\left(x_{j}\right)\right)\right] \\
& =\frac{2}{3}\left(30+\delta v_{i}\left(x_{i}\right)\right)+\frac{1}{3}\left(15+\delta v_{i}\left(x_{j}\right)\right)
\end{aligned}
$$

where $v_{i}\left(x_{j}\right)$ is the value function of the same agent $i$ when the state is $j$. With probability $1 / 3, i$ is the proposer and he can guarantee that $x_{i}$ is chosen; with probability $2 / 3$ an other agent (say $k$ ) is proposer and proposes $x_{k}$ : the proposal is accepted with probability $1 / 2$, with probability $1 / 2 x_{i}$ is implemented again. The continuation value at $x_{j}$ is for $i$ is can be computed in a similar way:

$$
v_{i}\left(x_{j}\right)=\frac{1}{6}\left(30+\delta v_{i}\left(x_{i}\right)\right)+\frac{5}{6}\left(15+\delta v_{i}\left(x_{j}\right)\right)
$$


Solving the system of equations (4)-(5) we have:

$$
v_{i}\left(x_{i}\right)=\frac{50-3 \delta}{(1-\delta)(2-\delta)}, v_{i}\left(x_{j}\right)=\frac{35-15 \delta}{(1-\delta)(2-\delta)}
$$

From these formulas it is easy to verify that the strategies described above induce a value function that is monotonically increasing in the agent's payoff. The following Proposition shows that not only these strategies are an equilibrium, but they are the unique symmetric Markov equilibrium:

Proposition 2. When the set of allocations are those in (2) and utilities are linear, there is a unique equilibrium in which players play myopic strategies.

The equilibrium strategies characterized in Proposition 2 imply a unique transition matrix. From a status quo $x_{i}$ the state remains at $x_{i}$ with probability $\frac{2}{3}$ and moves to a state $x_{j}$ with probability $\frac{1}{3}$, and never moves to $x_{0}$. From status quo $x_{0}$, the state remains at $x_{0}$ with probability 1 .

As in the NCW case, voting behavior predicted by proposition 3 is myopic, however this leads here to an important difference. In the NCW case, $(20,20,20)$ is defeated in any pairwise vote against any other allocation, but in the CW case, it will defeat any other allocation. Therefore, in the latter case, if the initial status quo is $(20,20,20)$, it will remain the status quo forever. Proposals of $(20,20,20)$, while off the equilibrium path in both the NCW and the CW case (except in the $\mathrm{CW}$ when it is the initial status quo) have a much different effect, since $(20,20,20)$ is an absorbing state in the CW game, but it is always defeated the NCW game. This implies drastically different dynamics in Markov QRE between the two tables, with Table 2 alternating between long epochs of "universal" regimes and "dictatorial" regimes, and Table 1 always yielding stable "majoritarian" regimes, where there is a random rotation of two players coalitions splitting the pie. The QRE dynamics are discussed in more detail in the results section.

\section{Laboratory Experiment}

We use controlled laboratory experiments to study behavior in these dynamic committee bargaining environments with endogenous status quo allocations. In our experimental design, we vary the discount factor and the set of feasible allocations. We conduct two sessions with discrete allocations, as in the theoretical section and three sessions with allocations with a very fine grid, as a finite approximation to the continuous-state divide the dollar game. We refer to the fine grid sessions as "continuous." In all sessions the sum of the three agents' allocations equals 60 . 


\section{IV.1 Procedures}

Discount factors were induced by a probabilistic endpoint. After each $t$, a fair die was rolled by the experimenter at the front of the room, and the game continued to period $t+1$ if and only if the die roll belonged to a pre-announced subset of the possible faces. For example, to implement $\delta=.75$, we rolled a twelve-sided die, and the game ended if and only if a 10,11, or 12 was rolled. ${ }^{10}$ If the die came up with a number less than 10 , the game proceeded to round $t+1$, with the status quo being determined by the majority rule winner in round $t$. In all except one session we used a discount factor of $\delta=.75$. The remaining session used $\delta=.83$ and a six-sided die.

The experiments were all conducted at the Princeton Laboratory for Experimental Social Science and used registered students from Princeton University. Each subject participated in exactly one session.

Each of the discrete allocation sessions was divided into two subsession, each of which lasted for 10 matches. Each match corresponded to one play of the infinitely repeated game, using the die-termination rule described above. ${ }^{11}$ The set of feasible allocations was different in the two subsessions. The two sets of allocations are exactly the ones previously described as the NCW and CW cases in (1) and (2).

In the continuous allocation sessions, proposals could be any non-negative integer division of the 60unit pie. Because this was a more difficult task, subjects took a long time deciding on their proposals. Consequently, we only ran 10 matches in each of the three continuous sessions.

\begin{tabular}{|c|c|c|c|c|}
\hline \multicolumn{5}{|c|}{ Table 3: Experimental Design } \\
\hline Session & $\boldsymbol{\delta}$ & Set of Feasible Allocations & & \# Subjects \\
\hline & & Matches 1 - 10 & Matches 11 - 20 & \\
\hline 1 & .75 & Table 1 & Table 2 & 9 \\
\hline 2 & .75 & Table 2 & Table 1 & 12 \\
\hline 3 & .83 & Continuous & & 12 \\
\hline 4 & .75 & Continuous & & 12 \\
\hline 5 & .75 & Continuous & & 12 \\
\hline
\end{tabular}

Instructions were read aloud and subjects were required to correctly answer all questions on a short comprehension quiz before the experiment was conducted. Subjects were also provided a summary sheet about the rules of the experiment which they could consult. The experiments were conducted via computers. $^{12}$

At the beginning of each match, subjects were randomly divided into committees of 3 members each. In each committee, members were assigned to be either Committee Member 1, Committee Member 2,

\footnotetext{
${ }^{10}$ In some sessions we used an 8 -sided die to implement $\delta=.75$. In the $\delta=.83$ session, we used a 6 -sided die.

${ }^{11} \mathrm{As}$ a result, there was a lot of variance in the length of the matches, which ranged from 1 round to 23 rounds.

${ }^{12}$ Sample instructions and the computer program used fro the experiment are available from the authors. The computer program used was an extension to the open source Multistage game software. See http://multistage.ssel.caltech.edu.
} 
or Committee Member 3 and this member assignment remained the same for all rounds of a match. An initial status quo was randomly chosen by the computer, using a uniform distribution of the set of feasible allocations. Initial status quo assignments were independent across matches and across committees.

After being informed of the initial status quo, each committee member was prompted by the computer to enter a "provisional proposal". After all members had entered a provisional proposal, one was selected at random to become the "active proposal". The active proposal was then voted on against the status quo, which was referred to as the "standing alternative". Whichever received more votes was the policy that was implemented in that round, and each member received earnings accordingly. After all committees had finished the round, a die was rolled to determine whether to continue. If the match continued, than the winning proposal in the previous round became the standing alternative for the new round. This continued until a specific die roll terminated the match.

This was repeated with the group membership shuffled randomly after each match. Each subject was paid the sum of his or her earnings over all rounds of all matches in cash at the end of the experiment. Average earnings were approximately $\$ 30$ (including a $\$ 10$ show up fee), with each session lasting about 90 minutes.

\section{Experimental Results}

We analyze the results separately for the discrete allocation sessions and the continuous allocation sessions.

\section{V.1 Discrete Allocation Sessions}

\section{V.1.1 State Transition Probabilities}

State transition probabilities provide a clear summary of the dynamics of outcomes since they provide a synthetic description of aggregate behavioral data on both proposal making and voting. ${ }^{13}$ The transitions and outcomes are summarized in Table 6 . For each table, the last row is obtained by summing each of the columns corresponding to the $t+1$ status quo. These frequencies give the overall outcome frequencies, excluding the initial round 0 status quos, which were decided randomly by the computer to start each match. Because the game is symmetric, it is useful to look at the transitions from the two key possible cases: when the status quo is the universal allocation or not. Figure (7) represents these transition probabilities.

\footnotetext{
${ }^{13}$ Note that this does not obviously follow, unless voting and proposal making strategies are stationary.
} 


\begin{tabular}{|c|c|c|c|c|c|}
\hline & \multicolumn{4}{|c|}{$\mathbf{S Q}(\mathbf{t}+\mathbf{1}) . C W$ committees } \\
\hline & & 30-15-15 & $15-30-15$ & $15-15-30$ & $20-20-20$ \\
\hline & 30-15-15 & $0.44(14)$ & $0.09(3)$ & $0.09(3)$ & $0.38(12)$ \\
\hline \multirow[t]{3}{*}{$S Q(t)$} & $15-30-15$ & $0.17(7)$ & $0.54(22)$ & $0.02(1)$ & $0.27(11)$ \\
\hline & $15-15-30$ & $0.17(5)$ & $0.03(1)$ & $0.53(16)$ & $0.27(8)$ \\
\hline & $20-20-20$ & $0.01(1)$ & $0.03(3)$ & $0.03(3)$ & $0.94(105)$ \\
\hline \multicolumn{2}{|c|}{ Freq (SQ) } & $0.13(27)$ & $0.13(29)$ & $0.11(23)$ & $0.63(136)$ \\
\hline & & \multicolumn{4}{|c|}{$\mathbf{S Q}(\mathbf{t}+\mathbf{1}) . \mathbf{N} C W$ committees } \\
\hline \multirow{5}{*}{$\operatorname{SQ}(\mathbf{t})$} & & $30-30-0$ & 30-0-30 & $0-30-30$ & $20-20-20$ \\
\hline & $30-30-0$ & $0.77(78)$ & $0.16(16)$ & $0.05(5)$ & $0.02(2)$ \\
\hline & $30-0-30$ & $0.13(12)$ & $0.79(73)$ & $0.05(5)$ & $0.02(2)$ \\
\hline & $0-30-30$ & $0.11(7)$ & $0.11(7)$ & $0.75(46)$ & $0.02(1)$ \\
\hline & $20-20-20$ & $0.19(7)$ & $0.16(6)$ & $0.14(5)$ & $0.51(19)$ \\
\hline \multicolumn{2}{|c|}{ Freq(SQ) } & $0.36(104)$ & $0.35(102)$ & $0.21(61)$ & 0.08 \\
\hline
\end{tabular}

Figure 6: The transition probability matrices in the CW and NCW games.

Several features of the outcome data are noteworthy. First, there is a striking difference between the outcomes of the two discrete treatments: in the NCW treatment, the universal outcome was the committee decision only 24 out of 291 times (8\%). In contrast, majoritarian outcomes prevailed nearly always: $92 \%$ of the time. For the CW treatment, this is reversed. There were 136 out of 215 (63\%) universal outcomes, while non-universal outcomes were chosen $37 \%$ of the time. The theoretical prediction of more universal outcomes in $\mathrm{CW}$ than NCW, therefore, is strongly supported by the data, and is significant at any conventional level.

Second, as also predicted by the theory, there is strong "persistence of regimes". For the CW treatment non-universal allocations should usually map into non-universal allocations and universal allocations map into universal allocations. This we find, with persistence rates of $70 \%$ in the case of the non-universal regime and $94 \%$ in the universal regime. In the NCW committees, the universal regime is not part of the equilibrium, so there should be less persistence, which is what we find: universal outcomes map into universal outcomes significantly less often (51\%) than in the CW committees. And we find nearly $100 \%$ persistence of majoritarian allocations in the NCW committees, as predicted. Hence, in all cases 

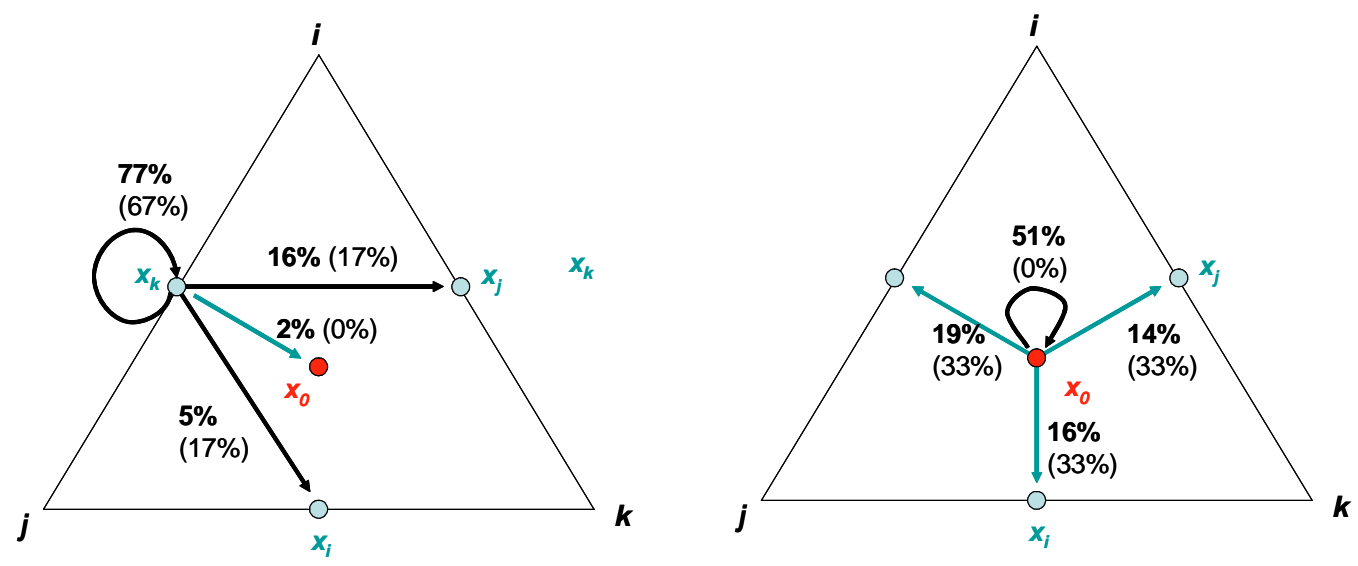

The NCW case
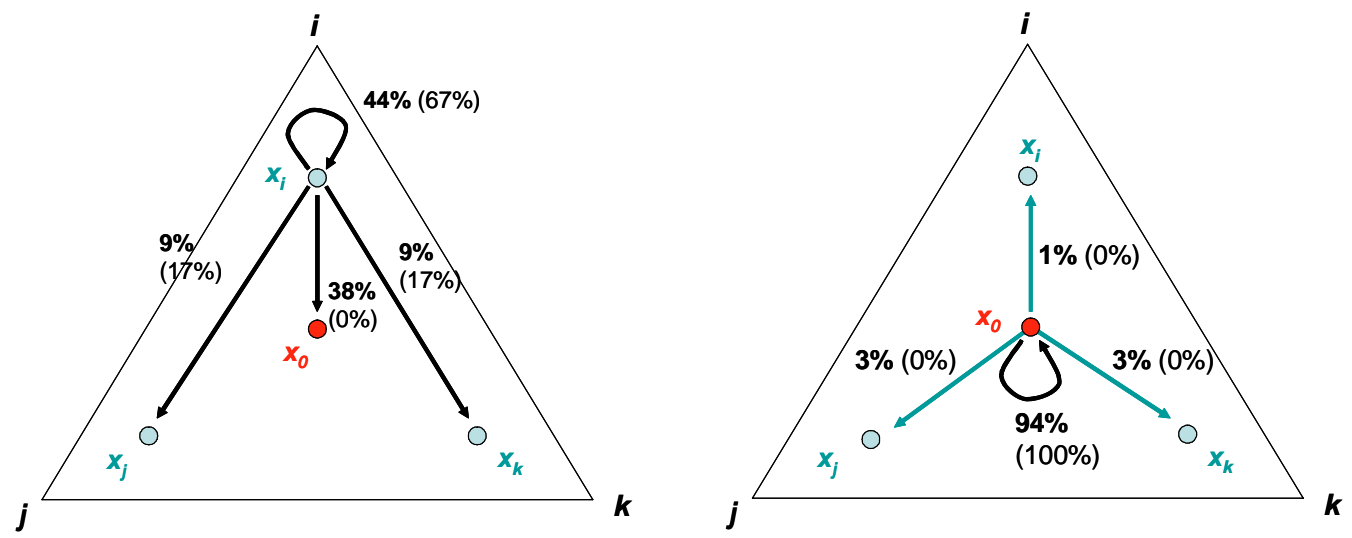

The CW case

Figure 7: Empirical transition probabilities for the NCW and $\mathrm{CW}$ game from the universal and majoritarian allocations. The numbers in parenthesis are the predictions of the equilibrium with linear utilities. 
except for non-universal outcomes in the NCW committees, universal or non-universal allocations are significantly more likely to persist than not, except in the case where they are not part of the Markov equilibrium (the case of universal allocation in NCW committees).

Tables (6), however, suggests an interesting systematic departure from equilibrium behavior. In particular, the non-universal regime is somewhat less stable than predicted in the $\mathrm{CW}$ committees, and universal allocations are somewhat more stable than predicted in the NCW committees. However, the latter is only $50 \%$ meaning that in $\mathrm{NCW}$ committees, universal allocations are as likely to be replaced by non-universal allocations as they are to persist; moreover, this is based on a very small number of observations, since universal allocations are rare $(8 \%)$ events for NCW committees.

To investigate the origin of these dynamic patterns, in the next two sections we decompose the determinants of the transition probabilities by analyzing in detail proposal and voting behavior. As we will show, the departure from the equilibrium observed above is mainly due to small deviations from equilibrium in proposal rather than voting strategies.

\section{V.1.2 Proposal Making and Outcomes}

Table 8 shows the aggregate proposal frequencies as a function of the status quo and the position of the player, for the $\mathrm{CW}$ (left) and NCW (right) treatments, respectively. The table is "anonymized" for data-pooling purposes, in the sense that a proposal of $(20,20,20)$ by member 1 when the status quo is $(30,30,0)$ is treated the same as a proposal of $(20,20,20)$ by member 2 when the status quo is $(0,30,30)$, and so forth. Furthermore, allocations that give an agent the equivalent share are combined. For example, in the $\mathrm{CW}$ treatment, observations of $15-15-30$ and $15-30-15$ are merged together for player 1 into the category "15". This anonymization leads to a very simple $3 \times 3$ matrix representation of the aggregate proposing data. For example, the entry 0.07 (7) in the CW table for row 30 column 20 indicates that $7 \%$ of subjects who receive 30 in the status quo under the CW treatment propose 20-20-20 (7 observations).

These tables show several features. First, subjects almost never offer to receive the lowest payoff (either 15 or 0 ). The rarity of these events $(2 \%)$ suggests that subjects understand the basic task, and such events are simply trembles that can be ignored.

Second, the proposal strategies in the NCW treatment closely track to the theoretical predictions. If the status quo is majoritarian, then all proposals are majoritarian more than $90 \%$ of the time (100\% is the prediction). In the relatively rare instances where the universal allocation is the status quo, that 


\begin{tabular}{llllllllll} 
CW & \multicolumn{4}{c}{ Proposal } & \multicolumn{3}{c}{ NCW } & \multicolumn{3}{c}{ Proposal } \\
& & $\mathbf{3 0}$ & $\mathbf{1 5}$ & $\mathbf{2 0}$ & & $\mathbf{3 0}$ & $\mathbf{0}$ & $\mathbf{2 0}$ \\
Status & $\mathbf{3 0}$ & $0.93(96)$ & $0.00(0)$ & $0.07(7)$ & Status & $\mathbf{3 0}$ & $0.94(479)$ & $0.01(7)$ & $0.04(22)$ \\
Quo & $\mathbf{1 5}$ & $0.57(117)$ & $0.06(13)$ & $0.37(76)$ & Quo & $\mathbf{0}$ & $0.89(226)$ & $0.01(3)$ & $0.10(25)$ \\
& $\mathbf{2 0}$ & $0.47(158)$ & $0.05(18)$ & $0.48(160)$ & & $\mathbf{2 0}$ & $0.70(78)$ & $0.01(1)$ & $0.29(32)$
\end{tabular}

Figure 8: Empirical Proposal Strategies

probability is more than $70 \%$.

Third, in contrast, the strategies in the CW treatment are not as close to the linear utility Markov equilibrium predictions. In one respect, it is consistent with the theory: just as in NCW, a favored committee member receiving 30 almost always (93\%) proposes to continue being the favored committee member. Both members receiving low payoffs in a non-universal status quo also usually (57\%) propose to be the new favored member, but propose the universal allocation slightly more than half as often (37\%). However, in the universal status quo, subjects are equally likely to propose their favorable allocation as they are to propose to stay at the universal outcome ${ }^{14}$. This is not in contrast with equilibrium behavior since in state $x_{0}$ a proposer is indifferent between proposals since $x_{0}$ defeats all proposals.

Thus, there are some sharp differences between the two treatments, the most important being the greater frequency of proposing 20 in the $\mathrm{CW}$ treatment, which is predicted by theory. However, in both the NCW treatment, subjects generally propose their short run favorable allocation, but the probability of making such a proposal varies with the state, being most likely if it already the current status quo, and least likely if the status quo is $20-20-20$.

Fourth, not shown in the table, there is statistically significant evidence of non-anonymous proposal making in the NCW treatment. This arises when there is a majoritarian status quo. The two coalition members are more than three times as likely ( $76 \%$ vs. $24 \%)$ to propose the same coalition, rather than proposing to switch partners. In contrast, no so such asymmetry is observed in the proposal strategy of the "out" member (or in the case of majoritarian proposals when the status quo is universal) with respect to which partner they propose to form a coalition with in a majoritarian outcome.

\footnotetext{
${ }^{14}$ The remaining $6 \%$ is accounted for by a few cases where a member proposed to be on the short end of an unequal allocation. This also happened in $5 \%$ of cases when the status quo was universal. In the NCW treatment there were also a few cases $(<5 \%)$ where a subject proposed an allocation where they would receive 0 . We have no logical explanation for these apparent "trembles".
} 


\begin{tabular}{lllll} 
CW & \multicolumn{4}{l}{ Standing Proposal } \\
& & $\mathbf{3 0}$ & $\mathbf{1 5}$ & $\mathbf{2 0}$ \\
Status & $\mathbf{3 0}$ & $0.44(27)$ & $0.00(42)$ & $0.06(34)$ \\
Quo & $\mathbf{1 5}$ & $1.00(42)$ & $0.42(96)$ & $0.96(68)$ \\
& $\mathbf{2 0}$ & $0.93(69)$ & $0.06(138)$ & $0.23(129)$
\end{tabular}

NCW Standing Proposal

$30 \quad 0 \quad 20$

$\begin{array}{lllll}\text { Status } & 30 & 0.25(360) & 0.03(118) & 0.17(30)\end{array}$

Quo $\quad 0 \quad 1.00(118) \quad 0.41(121) \quad 1.00(15)$

$20 \quad 0.86(50) \quad 0.00(25) \quad 0.28(36)$

Figure 9: Voting behavior. Entries are Pr\{vote for proposal\} (\# obs)

\section{V.1.3 Voting Decisions}

Voting decisions are overwhelmingly myopic and selfish for both treatments (meaning that agents vote for the alternative that offers the highest short run payoff), as predicted. Overall, in the two coarse grid sessions voters voted myopically $96 \%$ of the time (723/751). This is broken down in more detail, as follows. See Table 9.

In the CW treatment, when a member is faced with a choice a large share of a non-universal allocation (30) versus any other allocation (where their share would be either 20 or 15), they vote for the favorable allocation $96 \%$ of the time (180/187), and this does not depend on whether the favorable allocation was the status quo or the new proposal. When faced with a choice between the universal allocation and the smaller share of the non-universal allocation (15), a member voted for the universal allocation $97 \%$ of the time $(195 / 203) \cdot{ }^{15}$

In the NCW treatment, when faced with a choice between the alternative where they were out of the coalition and received 0 , versus any other alternative, the member voted for the other alternative $99 \%$ of the time $(292 / 296)$. When faced with a choice between receiving 30 in a majoritarian allocation versus receiving 20 in the universal allocation, they voted myopically for the majoritarian allocation $86 \%$ of the time $(56 / 65)$.

The similarity between the voting behavior in the two treatments is remarkable. The only real differences between the two tables is in the number of observations, not in the frequency of voting for one's myopically preferred alternative. Hence this leads us to conclude unambiguously that proposal behavior, not voting behavior, is what drives the differences in outcomes between the $C W$ and NCW treatments.

\footnotetext{
${ }^{15}$ This is strong evidence against any hypothesis of pro-social preferences playing a role in behavior here. Subjects in this experiment are clearly not motivated by concerns for fairness.
} 
NCW treatment

\section{P(proposal) P(yes)}

fitted status quo $=0 \quad 0.03$

data proposal $=0$

fitted status quo $=0$

data proposal $=\mathbf{2 0}$

fitted status quo $=0$

data proposal $=\mathbf{3 0}$

fitted status quo $=\mathbf{2 0}$

data proposal=0

fitted status quo $=\mathbf{2 0}$

data proposal $=\mathbf{2 0}$

fitted status quo $=\mathbf{2 0}$

data proposal $=\mathbf{3 0}$

fitted status quo $=\mathbf{3 0}$

data proposal $=0$

fitted status quo $=30$

data proposal $=\mathbf{2 0}$

fitted status quo $=\mathbf{3 0}$

data proposal $=30$

lambda

log Likelihood

$0.11 \quad 0.82$

0.15

1.00

0.86

0.85

0.01

0.01

0.18

0.00

0.19

0.31

0.81

0.68

0.81

0.84

0.02

0.02

0.03

0.03

0.35

0.19
0.17

0.63

0.90

0.19

$-867.33$
CW treatment

\section{P(proposal) $\mathbf{P ( y e s )}$}

status quo $=\mathbf{3 0} \quad 0.77$

proposal $=\mathbf{3 0}$

0.93

status quo $=\mathbf{3 0}$

proposal $=\mathbf{2 0}$

$0.14 \quad 0.21$

$0.07 \quad 0.06$

$\begin{array}{lll}\text { status quo }=30 & 0.09 & 0.10\end{array}$

proposal $=15 \quad 0.00 \quad 0.00$

$\begin{array}{lll}\text { status quo }=20 & 0.70 & 0.79\end{array}$

$\begin{array}{lll}\text { proposal=30 } & 0.47 & 0.93\end{array}$

status quo $=\mathbf{2 0} \quad 0.19$

proposal $=\mathbf{2 0} \quad 0.48$

status quo $=\mathbf{2 0} \quad 0.11 \quad 0.32$

$\begin{array}{lll}\text { proposal }=15 & 0.05 & 0.06\end{array}$

status quo=15 $\quad 0.74 \quad 0.89$

$\begin{array}{lll}\text { proposal }=30 & 0.57 & 1.00\end{array}$

$\begin{array}{lll}\text { status quo }=15 & 0.18 & 0.68\end{array}$

$\begin{array}{lll}\text { proposal }=20 & \mathbf{2 0} & 0.37\end{array}$

status quo $=15 \quad 0.09$

proposal=15 $\quad 0.06$

0.24

$-905.05$

Figure 10: Figure 11. MLE fitted choice Probabilities compared with actual data.

\section{V.1.4 Quantal Response Equilibrium: Markov Logit Equilibrium (MLE)}

As discussed earlier, because there are two regimes in the CW treatment, a quantal response equilibrium analysis will produce a somewhat different dynamic compared to the Markov Nash equilibrium. In the Markov Nash equilibrium, depending on the randomly assigned initial status quo, each committee will find itself stuck forever in exactly one regime, either the regime of the static condorcet winner (where the universal outcome occurs every round) or the rotation regime, where outcomes randomly rotate around the three non-universal allocations That is, they "lock in" on one regime from the very start and stay there forever, and this is entirely determined by the initial status quo. In a quantal response equilibrium, stochastic choice will result in long run alternation between the two regimes.

Table 10 reports the fitted and actual choice probabilities for all the different behavior strategies of 
the players, under the assumption of anonymity (e.g., the probability voter 1 votes for $(30,15,15)$ over $(20,20,20)$ equals the probability voter 2 votes for $(15,30,15)$ over $(20,20,20)$, etc.). The fitted choice probabilities are the MLE choice probabilities at the maximum likelihood value of lambda, estimated separately for the two treatments. ${ }^{16}$ The estimated choice probabilities track the data reasonably well. The following figure presents a scatter plot of the predicted and actual choice probabilities, and also shows the regression line, which has slope close to 1 and intercept close to 0 , and $R^{2}=.85$.

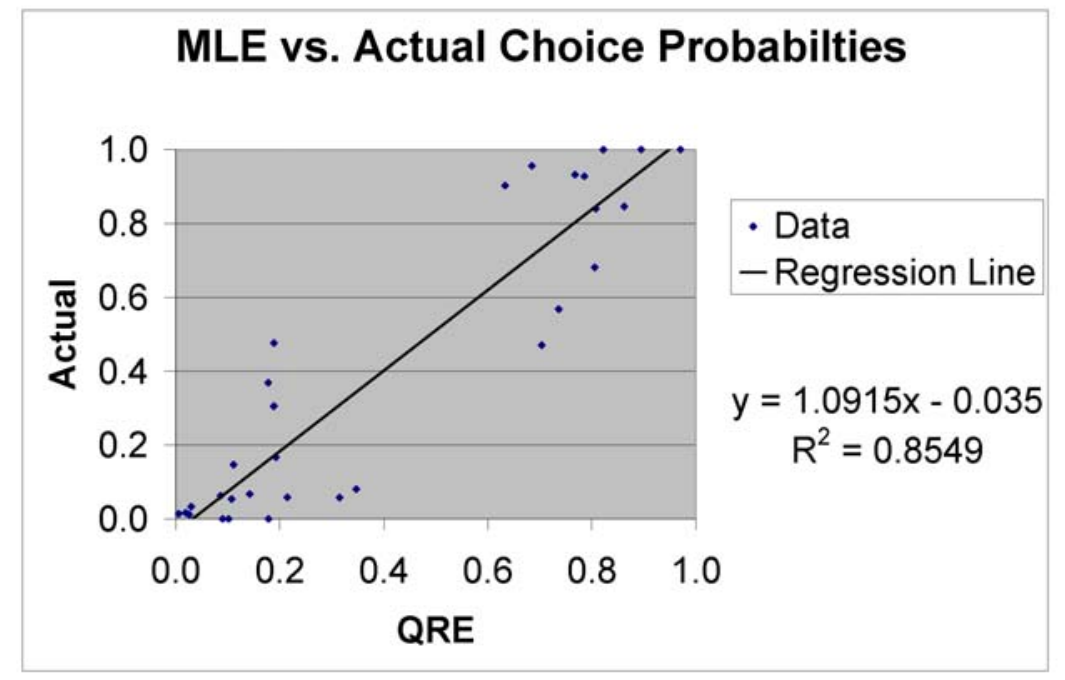

\section{V.2 Continuous Allocation Sessions}

We analyze continuous Session 1 separately from Sessions 2 and 3, because the discount rate was different. Several interesting comparisons emerge, in spite of the fact that the Markov equilibria are identical with linear preferences. As we will see, these many of these differences can be explained extending the analysis to Markov QRE and considering concave utilities.

\section{V.2.1 Empirical distribution and transition Probabilities}

Table 11 shows the transition frequencies, in percentages for each status quo, using the 7-region grid described in Section III.1.2 (see Figure (1)). ${ }^{17}$ Regions D1, D2, and D3 are the dictatorial regions; M12, M13, and M23 are the majoritarian regions, and $\mathrm{U}$ is the universal region. The top half is for impatient

\footnotetext{
${ }^{16}$ Recall that each treatment obtained data from two separate sessions, one in which it was the first subsession and another where it was the second subsession. Lambdas estimated separately for each session are not significantly different.

${ }^{17}$ The actual implementation in the laboratory was finite, with 1891 possible states (proposals to divide 60 into three nonnegative integer allocations). For computational reasons, the Markov QRE, and the Nash equilibrium benchmarks were computed assuming the coarser grid described in Section III.1.1 where the unit of measure is 5 (which implies 91 states). To compare the results, the states in the experimental state space were aggregated associating each of them to the closest state (in euclidean norm) in the coarser 91-state space. Over $90 \%$ of proposed allocations in the experiment, however, are divisible by 5 , so the impact of this approximation is minimal.
} 
committees and the bottom half for patient committees. The last row in each half gives the relative frequencies of outcomes in each region. The left panel of Figure 15 represents the empirical transition probabilities (as done in Figure 3).

These tables yield the following results. From the last rows of the upper and lower half of Table 11, this clearly refutes the Markov equilibrium prediction based on linear utilities that the committees outcomes will approximate a rotating dictatorship (Table 2 and Figure 3A). We observe outcomes in the $\mathrm{D}$ regions only $10 \%$ of the time in the patient committees, and only $26 \%$ of the time in impatient committees. It is worth noting, however, that there is still a lot of persistence to dictatorial outcomes. Conditional on the status quo being in a $\mathrm{D}$ region, the outcome in the next period is almost twice as likely to be in one of these regions compared to non-D outcomes, with this persistence strongest for impatient committees. The reason so few outcomes are observed in these regions overall is that they are only occasionally reached from any other regions (less than $10 \%$ of the time).

In the patient committees, the remaining outcomes are divided equally between majoritarian outcomes and the universal outcome (approximately $45 \%$ of the time each). The transition matrix gives some indication of the dynamics of the patient committees. The universal outcomes function nearly, but not quite, as an absorbing state. The empirical probability of moving away from $\mathrm{U}$ is only $12 \%$. In the linear utility equilibrium, this probability is $100 \%$, and it should be defeated by majoritarian proposals. ${ }^{18}$ Indeed the latter property of equilibrium is observed to an extent in our data: i.e., when $\mathrm{U}$ is defeated, it is almost always by a majoritarian (2-person coalition) proposal. Majoritarian status quos are less stable than $\mathrm{U}$, with the probability of transitioning to a new region being approximately $50 \%$. The theoretical (with linear utilities) probability of a majority status quo being defeated is $100 \%$, and it should be defeated only by dictatorial proposals. In fact, we find the opposite: when a M status quo is defeated, it is usually by $\mathrm{U}$, not by a dictatorial proposal. The $\mathrm{D}$ status quos are by far the least stable, in contrast to the linear utility model, which predicts them to be the most stable (being defeated only $33 \%$ of the time, always by another D outcome). In the experiment, they are defeated almost $2 / 3$ of the time - essentially whenever one of the non-dictators is chosen as the proposer.

The distribution of outcomes for the impatient committees is different. D outcomes are somewhat more common, but still occur only slightly more than one-quarter of the time. Outside the D regions,

\footnotetext{
${ }^{18}$ For, example if the status quo is $(20,20,20)$, voter 1 's equilibrium equilibrium proposal strategy is to mix $50 / 50$ between $(39,21,0)$ and $(39,0,21)$.
} 


\begin{tabular}{|c|c|c|c|c|c|c|c|c|}
\hline \multirow[t]{2}{*}{$\delta=.83$} & & \multicolumn{7}{|c|}{ Status Quo $(\mathbf{t}+\mathbf{1}) \cdot \delta=.83$} \\
\hline & & D1 & D2 & D3 & M12 & M13 & M23 & U \\
\hline Status & D1 & $0.33(3)$ & $0.22(2)$ & $0.11(1)$ & $0.00(0)$ & $0.00(0)$ & $0.33(3)$ & $0.00(0)$ \\
\hline \multirow[t]{6}{*}{ Quo (t) } & D2 & $0.14(1)$ & $0.14(1)$ & $0.14(1)$ & $0.00(0)$ & $0.43(3)$ & $0.14(1)$ & $0.00(0)$ \\
\hline & D3 & $0.06(1)$ & $0.06(1)$ & $0.44(8)$ & $0.33(8)$ & $0.00(0)$ & $0.00(0)$ & $0.11(2)$ \\
\hline & M12 & $0.02(1)$ & $0.06(3)$ & $0.00(0)$ & $0.5528)$ & $0.14(7)$ & $0.12(6)$ & $0.12(6)$ \\
\hline & M13 & $0.06(3)$ & $0.00(0)$ & $0.04(2)$ & $0.10(5)$ & $0.67(34) \quad c$ & $0.08(4)$ & $0.06(3)$ \\
\hline & M23 & $0.00(0)$ & $0.04(1)$ & $0.11(3)$ & $0.11(3)$ & $0.18(5)$ & $0.39(11)$ & $0.18(5)$ \\
\hline & $\mathbf{U}$ & $0.00(0)$ & $0.01(1)$ & $0.00(0)$ & $0.07(9)$ & $0.02(3)$ & $0.02(2)$ & $0.88(107)$ \\
\hline \multicolumn{2}{|c|}{ Freq(SQ) } & 0.03 & 0.03 & 0.05 & 0.18 & 0.18 & 0.09 & 0.43 \\
\hline \multirow{2}{*}{\multicolumn{2}{|c|}{$\delta=.75$}} & \multicolumn{7}{|c|}{ Status Quo (t+1). $\delta=.75$} \\
\hline & & D1 & D2 & D3 & M12 & M13 & M23 & $\mathbf{U}$ \\
\hline Status & D1 & $0.33(7)$ & $0.10(2)$ & $0.14(3)$ & $0.05(1)$ & $0.00(0)$ & $0.33(7)$ & $0.05(1)$ \\
\hline \multirow[t]{6}{*}{ Quo (t) } & D2 & $0.18(3)$ & $0.18(3)$ & $0.35(6)$ & $0.18(3)$ & $0.12(2)$ & $0.00(0)$ & $0.00(0)$ \\
\hline & D3 & $0.13(5)$ & $0.16(6)$ & $0.42(16)$ & $0.16(6)$ & $0.00(0)$ & $0.00(0)$ & $0.13(5)$ \\
\hline & M12 & $0.05(4)$ & $0.10(8)$ & $0.00(0)$ & $0.31(25)$ & $0.27(22)$ & $0.25(20)$ & $0.02(2)$ \\
\hline & M13 & $0.06(4)$ & $0.00(0)$ & $0.03(2)$ & $0.22(15)$ & $0.46(31)$ & $0.18(12)$ & $0.04(3)$ \\
\hline & M23 & $0.00(0)$ & $0.11(7)$ & $0.17(11)$ & $0.21(13)$ & $0.22(14)$ & $0.27(17)$ & $0.02(1)$ \\
\hline & $\mathbf{U}$ & $0.00(0)$ & $0.00(0)$ & $0.00(0)$ & $0.21(11)$ & $0.28(15)$ & $0.09(5)$ & $0.42(22)$ \\
\hline \multicolumn{2}{|c|}{ Freq(SQ) } & 0.07 & 0.08 & 0.11 & 0.22 & 0.25 & 0.18 & 0.10 \\
\hline
\end{tabular}

Figure 11: State Transition Probabilities.Continuous data converted to 7-region grid.

outcomes are significantly more likely to lie in M than in U (64\% vs. 10\%), compared with the patient committee outcomes where the difference is not significant (45\% vs. $43 \%$ ). The reason for a difference in transition probabilities between the treatments is that $\mathrm{U}$ region is essentially absorbing in the patient committees, while in the impatient committees, a status quo of $U$ is usually defeated immediately by a $\mathrm{M}$ proposal. The $\mathrm{D}$ and $\mathrm{M}$ regions all have strong persistence for impatient committees, stronger than in the patient committees. Conditional on being in a $\mathrm{D}$ region, the empirical probability of staying in some $\mathrm{D}$ region is $67 \%$ (for the patient committees it was $56 \%$ ). Conditional on being in a $\mathrm{M}$ region, the empirical probability of staying in that region or transitioning to a different 2-person coalition is over $80 \%$ (for the patient committees, it was $79 \%$, so the difference in this case is not significant). In contrast, the $\mathrm{U}$ region is unstable: the probability of transitioning away from $\mathrm{U}$ is nearly $60 \%$, compared to $12 \%$ in patient committees.

We now decompose the data into proposal and voting behavior. To compare the evidence with in the discrete states treatment discussed earlier, it is useful to condense the 7 regions of Figure 1 into 5 regions by combining regions that are "anonymously equivalent" from the point of view of a member in 


\begin{tabular}{|c|c|c|c|c|c|c|c|}
\hline & & \multicolumn{6}{|c|}{ Provisional Proposal. $\delta=.83$} \\
\hline & & D & DX & M & $\mathrm{MX}$ & $\mathbf{U}$ & \#OBS \\
\hline Status & D & 0.88 & 0.00 & 0.09 & 0.00 & 0.03 & 34 \\
\hline \multirow[t]{6}{*}{ Quo } & DX & 0.34 & 0.01 & 0.51 & 0.00 & 0.13 & 68 \\
\hline & M & 0.19 & 0.00 & 0.66 & 0.01 & 0.14 & 260 \\
\hline & $\mathrm{MX}$ & 0.00 & 0.08 & 0.71 & 0.00 & 0.21 & 130 \\
\hline & $\mathbf{U}$ & 0.01 & 0.01 & 0.33 & 0.00 & 0.66 & 366 \\
\hline & & \multicolumn{6}{|c|}{ Provisional Proposal. $\delta=.75$} \\
\hline & & D & DX & M & $\mathrm{MX}$ & $\mathbf{U}$ & \#OBS \\
\hline Status & D & 0.78 & 0.00 & 0.18 & 0.01 & 0.03 & 76 \\
\hline \multirow[t]{4}{*}{ Quo } & DX & 0.58 & 0.00 & 0.35 & 0.01 & 0.07 & 152 \\
\hline & M & 0.28 & 0.01 & 0.65 & 0.00 & 0.06 & 422 \\
\hline & $M X$ & 0.00 & 0.05 & 0.90 & 0.00 & 0.05 & 211 \\
\hline & $\mathbf{U}$ & 0.03 & 0.01 & 0.78 & 0.01 & 0.18 & 159 \\
\hline
\end{tabular}

Figure 12: Proposal probabilities, conditional on Status Quo.

a symmetric equilibrium. For example, for player 1, D2 and D3 are combined and $M 12$ and $M 13$ are combined. We have relabeled the 5 regions: $D, D X, M, M X, U$. If we take agent $i$ as a reference, $D$ is the region where a proposer $i$ receives the most and corresponds to $D i$ in the 7 region partition; $D X$ is composed by $D j$ and $D k$ for $j, k \neq i ; M$ is $M i j$ and $M i k ; M X$ is $M j k$; and $U$ is the same as in the seven region partition.

\section{V.2.2 Proposal making and voting behavior}

Table 12 shows the aggregate proposing behavior for continuous committees for each status quo. The top half of the table is for the "patient" committees $(\delta=.83)$. The bottom half of the table is for the "impatient" committees $(\delta=.75)$. The entries in the table are the frequencies of proposal in that category, given the status quo, pooling across all committees, rounds, and members.

There are several notable features. First, as in the coarse grid data, subjects almost never propose to have the smallest allocation. That is, we see essentially no observations of a subject proposing an allocation in DX or in MX. Second, if a member is receiving a dictatorial allocation (i.e., the status quo is in $\mathrm{D}$ ), then they almost always propose to stay in $\mathrm{D}$ - as predicted by the theory, and also consistent with the evidence from the discrete treatment. Interestingly, U proposals are almost never made by a subject in the $\mathrm{D}, \mathrm{DX}$, or $\mathrm{M}$ states. There is a lot of persistence of proposals in both the $\mathrm{M}$ and the $\mathrm{D}$ 
regions in both kinds of committees; subjects usually propose to stay in the same region, about $2 / 3$ of the time.

In the patient committees, when the status quo is in the $\mathrm{U}$ region, $\mathrm{U}$ proposals are proposed $2 / 3$ of the time and $\mathrm{M}$ proposals are made $1 / 3$ of the time. While the linear utility equilibrium predicts almost all proposals in the $\mathrm{U}$ regions should be outside $\mathrm{U}$, this is no longer true for $\gamma>0$. Depending on the concavity of the utility function the equilibrium proposal when the status quo is in $U$ can be in either region $\mathrm{U}$ or $\mathrm{M}$. In the DX region (as seen in the analysis of the cases with $\gamma=0$ and $\gamma=0.95$ of Section III.1.2), the Nash proposals are either in D or M, which is true $93 \%$ of the time in our data. For MX status quos we never see $\mathrm{D}$ proposals, which is consistent with equilibrium behavior because $\mathrm{D}$ proposals will always be defeated. In the $\mathrm{M}$ region, the non-M proposals are equally split between $\mathrm{D}$ and $\mathrm{U}$.

The proposing behavior is different in the impatient committees compared to the patient committees, especially in the $\mathrm{U}$ region. In impatient committees, $\mathrm{U}$ allocations are rarely proposed at any status quo. Intuitively, this makes sense because the $U$ allocation only is valuable under two conditions, first utilities must be sufficiently concave, and second, the continuation probability must be high enough to make the value of an equal share tomorrow be higher than a more than equal share today. To see this, consider the case where $\delta$ is very small, so that value is determined almost entirely by your current share in the allocation. Then you would most prefer $\mathrm{D}(\approx 60)$, next most prefer $\mathrm{M}(\approx 30)$, and $\mathrm{U}(\approx 20)$ is least preferred. This is reflected in the data where over $90 \%$ of proposals are in M or D. Clearly the M proposals are most likely in the MX region, because these are the only proposals that can make the proposer better off and also make one other coalition partner better off.

Voting decisions are somewhat less myopic ${ }^{19}$ in the continuous allocation committees than in the CW and NCW committees with only 4 states. While $96 \%$ of voting decisions were myopic in those coarse-grid treatments, only $83 \%$ of voting decisions were myopic for $\delta=.83$ and $92 \%$ of the decisions are myopic for $\delta=.75$. Still, an overwhelming fraction of voting decisions are myopic. Part of the lower frequency of myopic decision making in the continuous allocation committees is probably attributable to the fact that often subjects were faced with a choice between two alternatives that were quite close in terms of their share of the allocation. In the coarse grid treatments, no such pairs exist; a voter is either completely indifferent or receives a significantly higher share in one of the alternatives. But there is another factor that is also important. In contrast to the discrete sessions, the value functions in the

\footnotetext{
${ }^{19}$ By myopic we mean voting in short run self interest.
} 


\begin{tabular}{lllllll}
\multicolumn{7}{c}{ Active Proposal. $\delta=.83$} \\
Status & D & $0.91(11)$ & $0.00(7)$ & $0.00(2)$ & $0.08(12)$ & $0.50(2)$ \\
Quo & DX & $1.00(7)$ & $0.83(29)$ & $1.00(26)$ & $0.50(2)$ & $1.00(4)$ \\
& M & $0.89(18)$ & $0.11(18)$ & $0.78(135)$ & $0.09(47)$ & $0.45(42)$ \\
& MX & $(0)$ & $0.83(18)$ & $1.00(47)$ & $0.57(44)$ & $1.00(21)$ \\
& U & $0.75(4)$ & $0.13(8)$ & $0.66(70)$ & $0.09(35)$ & $0.86(249)$ \\
& & & & & & \\
Status & D & $0.91(22)$ & $0.00(27)$ & $0.40(5)$ & $0.13(16)$ & $0.33(6)$ \\
Quo & DX & $1.00(27)$ & $0.70(71)$ & $0.97(37)$ & $0.60(5)$ & $1.00(12)$ \\
& M & $1.00(39)$ & $0.05(41)$ & $0.80(204)$ & $0.09(116)$ & $0.32(22)$ \\
& MX & $1.00(1)$ & $0.92(39)$ & $1.00(116)$ & $0.41(44)$ & $1.00(11)$ \\
& $\mathbf{U}$ & $1.00(1)$ & $0.00(2)$ & $0.84(90)$ & $0.04(45)$ & $0.67(21)$
\end{tabular}

Figure 13: Prob\{Vote for Proposal\} (\# obs)

continuous treatments are not monotone increasing in a members own allocation.

The top half of Table 13 shows the aggregate voting behavior for (status quo, active proposal) pairs with patient committees for each status quo, using the 5-region grid. The entries in the table are the empirical fraction of yes votes (in favor of the active proposal), pooling across all committees, rounds, and members. The bottom table shows the relative frequencies of the active proposals for each status quo category.

There are several results that follow from these tables. First, members almost always vote for D outcomes over any alternative. Second, members tend to vote for majoritarian outcomes, against any alternative, except when the status quo is being the dictator. Even in that case, members vote for the majoritarian outcome $1 / 3$ of the time, but these kinds of elections are rare events. Third, members tend to vote for $\mathrm{U}$ over other alternatives, except majoritarian outcomes, where the two members of the majoritarian coalition usually vote against $\mathrm{U}$. Indeed, the predominance of $\mathrm{U}$ votes in the $\mathrm{U}$ state accounts the bulk of the non-monotonic voting behavior in the session.

The bottom half of Table 13 shows the aggregate voting behavior for (status quo, active proposal) pairs in impatient committees for each status quo, again using the 5-region grid. The results are much the same as the voting behavior in patient committees. The only difference is that impatient committees tended to vote for $\mathrm{M}$ proposals over $\mathrm{U}$ proposals with slightly greater frequency, but the difference is rather 
small in magnitude. This finding is similar to what we observed in the discrete allocation committees, where the main differences in behavior across treatments was in proposal behavior, not voting behavior.

\section{V.3 Comparison with theory: QRE and Concave Utilities}

For the continuous allocation environments, the predictions of the Markov equilibrium with linear preferences $(\gamma=0)$ is clearly rejected by the data, for at least three reasons. First, in both treatments the dictatorial outcomes are rarely observed ( $22 \%$ in the .75 treatment and $12 \%$ in the .83 treatment). Over $80 \%$ of outcomes are either Majoritarian or Universal. Second, as it can be seen by comparing Figure 3 with the left panel of Figure 15 and 16, the transition probabilities are quite different for the two sessions: the .83 treatment has many more $\mathrm{U}$ observations and more persistence in the $\mathrm{U}$ state but less persistence in the D and M states. But the Markov equilibrium with linear preferences predicts no treatment effect. Third, there are many transitions (and proposals) that are predicted never to happen according to the Markov equilibrium with linear preferences.

A possible explanation for this finding is that agents have altruistic preferences. This hypothesis, however, seems contradicted by voting behavior, which is predominantly myopic and selfish, and proposing behavior: since, as we have seen in the previous section, proposers seem to take advantage of proposal power whenever the status quo allows them to do it.

Based on the theoretical analysis earlier in the paper, we can suggest an alternative explanation for the findings which is based on more standard assumptions on utility: that individuals have strictly concave utility functions. To test this hypothesis, we estimate the value of $\gamma$ in each treatment, and also estimate a constrained value of $\gamma$, assuming it to be the same in both treatments, and assuming it to be the same across subjects. In order to obtain such an estimate, we use the Logit equilibrium as a structural model of the errors, and therefore simultaneously estimate $\lambda$ and $\gamma$. The QRE model is a natural one to use for the error structure to estimate $\gamma$, because evidence from past experiments indicate a significant stochastic component of choice, which is correlated with equilibrium expected payoffs. Because the stochastic choice affects expected payoffs, it will generally have additional equilibrium effects. The estimation is done using standard maximum likelihood methods. Using the path following algorithm used earlier to compute Nash equilibria, we trace out the logit solution to the game; that is, we trace out a unique connected family

of MLEs, $\{\rho(\lambda, \gamma), \sigma(\lambda, \gamma)\}$ for increasing values of lambda and $\gamma$ ranging from 0 to 1 . This defines a likelihood function $L(\widehat{\rho}, \widehat{\sigma} ; \gamma, \lambda)$, where $(\widehat{\rho}, \widehat{\sigma})$ are the observed proposal and voting choice frequencies in 


$\begin{array}{cccc}\delta & \hat{\gamma} & \hat{\lambda} & -\operatorname{In} \mathbf{L} \\ .83 & 0.70 & 8.01 & 3827 \\ .75 & 0.40 & 1.20 & 3792 \\ \text { Pooled } & 0.50 & 1.68 & 7643\end{array}$

Figure 14:

the data, using the 91 state grid defined earlier in the paper.

Table 14 below gives the results of the estimation for the continuous sessions. In both treatments, the concavity parameter is highly significant $(p<.001)$. The estimates we obtain are also in the same range (between 0.40 and 0.70 ) as concavity estimates from many other sources of data, including auction experiments (Goeree, Holt, and Palfrey 2002), to abstract game experiments (Goeree, Holt, and Palfrey 2003), lottery choice experiments (Holt and Laury (2002)), and field data from auctions (Campo, Guerre, Perrigne, and Vuong 2000). The Chi-square test shows a significant difference between $(\widehat{\lambda}, \widehat{\gamma})$ for the two treatments. However, the differences between the constrained (pooled) estimates and the separate estimates in terms of the fit to voting and proposing behavior are small. The improvement in likelihood with the separate estimates is less than one-third of one percent, compared to the constrained (pooled) estimates. While the estimates of $\widehat{\boldsymbol{\lambda}}$ appear quite different across the two treatments, this is exaggerated by a ridge in the likelihood functions, where slightly higher values of $\widehat{\gamma}$ lead to much higher estimates of $\widehat{\boldsymbol{\lambda}}$, especially with the .83 data, but with virtually no change in the likelihood function. For example, in the .83 data, $-\mathbf{l n} \mathbf{L}[\widehat{\gamma}=.65, \widehat{\boldsymbol{\lambda}}=3.01]=3832$ and $-\ln \mathbf{L}[\widehat{\gamma}=.70, \widehat{\boldsymbol{\lambda}}=8.01]=3827$.

Figure 15 compares the empirical transition matrices for the two treatments, using the 7 -state grid with the theoretical transition matrices implied at the estimated values of $\widehat{\gamma}$ and $\widehat{\boldsymbol{\lambda}}$. The theoretical transitions for this coarser grid are derived in the following way. First, using the 91-state grid, the estimated parameters directly imply estimated proposal and voting strategies in the $(\widehat{\gamma}, \widehat{\boldsymbol{\lambda}})$ Markov logit equilibrium. We then use those estimated proposal and voting strategies to obtain a $91 \times 91$ transition matrix, for the states shown in Figure 1. In order to condense this into a $7 \times 7$ transition matrix, we need to weight the states within each of the 7 coarsened states according to the probability those states would theoretically occur. The apparently obvious way to do this by using the stationary distribution implied by the theoretical transition matrix turns out to be incorrect, because in our experiment we used 

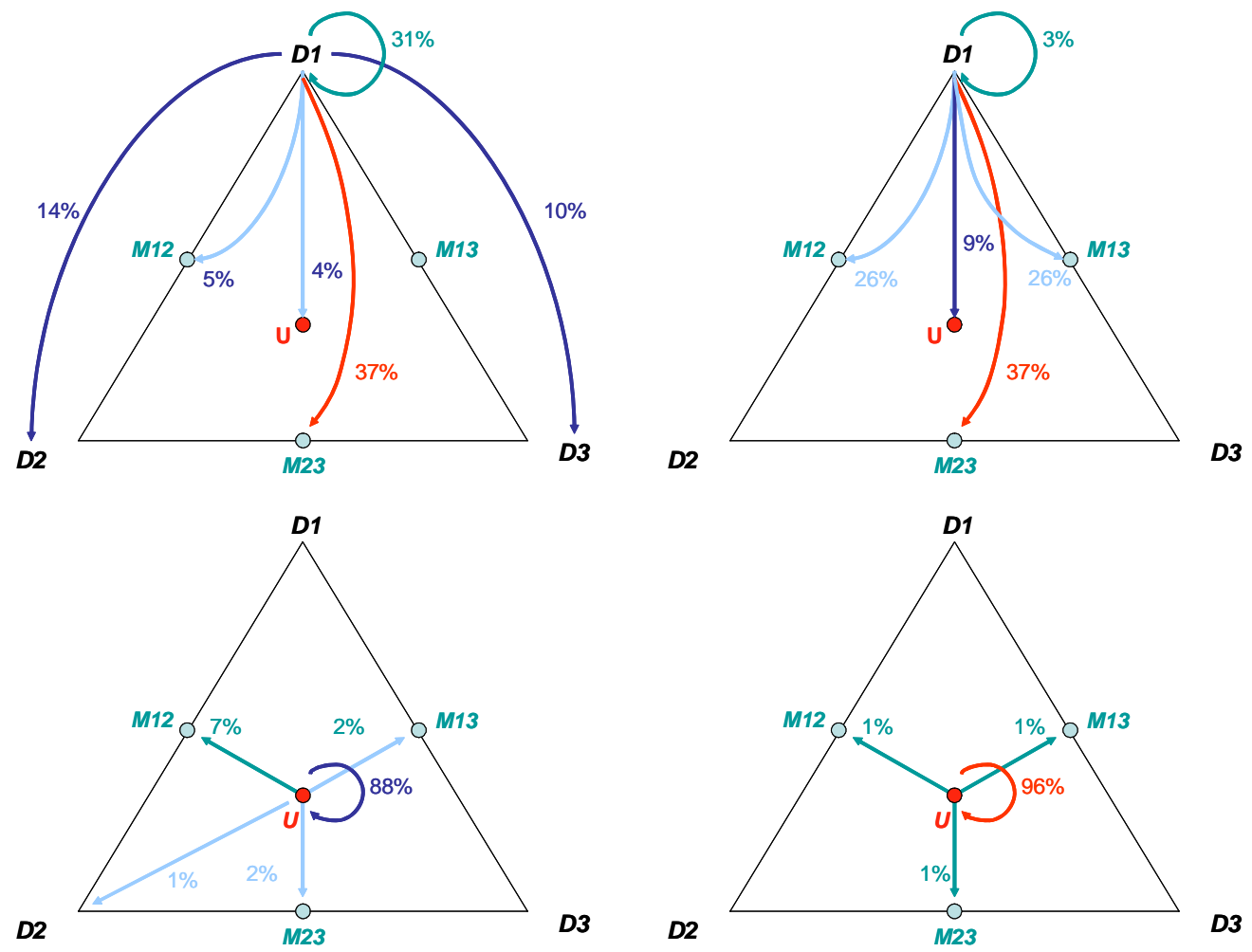

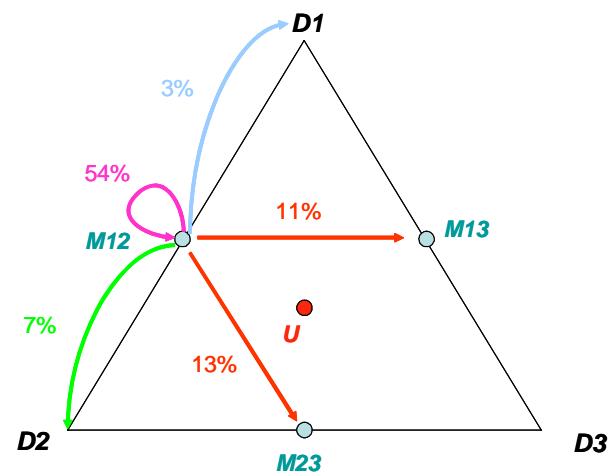

Empirical: $d=0.83$

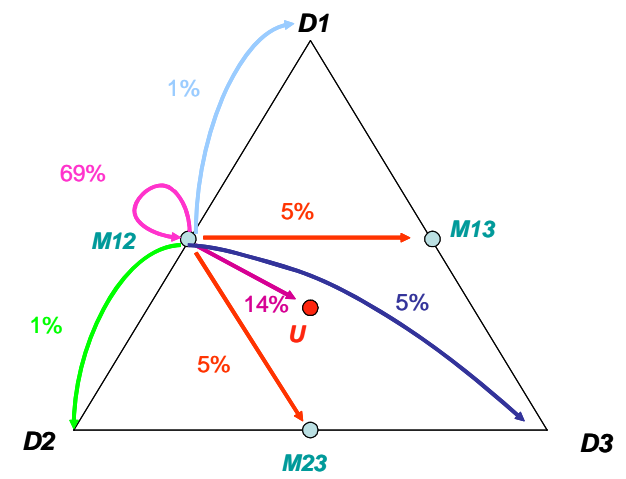

Weighted QRE: $d=0.83$

Figure 15: 
a random stopping rule, rather than playing the game an infinite number of times. Hence the actual distribution is heavily influenced by the initial status quo allocations, which were uniformly distributed on the simplex. Thus, we compute the expected distribution, given that we start round 1 with a uniform distribution over the allocations, and then compute the implied distribution over future allocations, given the stationary stopping rule (either .83 or .75 ). These weights are then used to coarsen the transition matrix to $7 \times 7 .^{20}$

There are some apparent similarities between the theoretical transition matrices and the empirical ones, but also some differences. First, for both the theoretical and empirical transitions, the status quo has a lot of persistence in all regions of both treatments, with the single exception being the $\mathrm{D}$ regions of the .83 treatment. For example, in .75 treatment, for the theoretical transitions at the $(\widehat{\lambda}, \widehat{\gamma})$ estimates, the probability of staying in a D region, give the status quo is a D outcome is nearly $70 \%$; it is $60 \%$ for the $\mathrm{M}$ regions; and $56 \%$ for the $\mathrm{U}$ region. The corresponding empirical probabilities are $66 \%, 80 \%$, and $42 \%$, respectively. For the .83 treatment, these theoretical persistence probabilities for the $\mathrm{M}$ and $\mathrm{U}$ regions are $79 \%$ and $96 \%$, respectively compared with the empirical findings of $78 \%$ and $88 \%$, respectively. For the exceptional case of the $\mathrm{D}$ regions in the .83 treatment, the theoretical persistence probability is $3 \%$ and the observed finding is $55 \%$. However, this latter case is based on very few observations, as we observed observations in the $\mathrm{D}$ regions a meager $10 \%$ of the time.

There are also similarities between the empirical and theoretical non-persistent transitions $(\mathrm{D} \rightarrow \mathrm{M}$ or $\mathrm{U}, \mathrm{M} \rightarrow \mathrm{D}$ or $\mathrm{U}$, and $\mathrm{U} \rightarrow \mathrm{D}$ or $\mathrm{M})$. For the .83 committees, when the status quo transitions out of a $\mathrm{D}$ region, we find that when the status quo is $\mathrm{D}$ it goes to an $\mathrm{M}$ allocation $88 \%$ of the time, identical to the theoretical transition probability from $\mathrm{D}$ to $\mathrm{M}$; when the status quo leave the $\mathrm{U}$ region, we observe it going to an M region over $90 \%$ of the time, where the theoretical prediction is $100 \%$ ' from an M region, we observe it going to both $\mathrm{U}$ and $\mathrm{D}$, but somewhat more frequently to $\mathrm{U}$, consistent with the theoretical transition probabilities. For the .75 committees the non-persistent transitions also track the theoretical transitions reasonably well: from $\mathrm{D}$ we observe transitions to $\mathrm{M}$ three times more often than transitions to $\mathrm{U}$, where the theoretical ration is two to one; from $\mathrm{M}$, transitions to $\mathrm{D}$ account for $81 \%$ of the non-persistent transitions in our data, compared the a theoretical transition probability of $79 \%$; from $\mathrm{U}$, transitions to $\mathrm{M}$ are more likely than transitions to $\mathrm{D}$ theoretically, and we observe this in the data.

\footnotetext{
${ }^{20}$ To avoid clutter, the figure only shows the transitions from D1 (top), U (middle), and M12 (bottom). By symmetry, the theoretical transitions (right half of figure) from D2 and D3 are identical to the one illustrated for D1; transitions from M13 and M23 are identical to the one illustrated for M12. The empirical transitions illustrated from D1 (top left) represent the average across all three D regions; similarly for the empirical transitions from M12 (bottom left).
} 

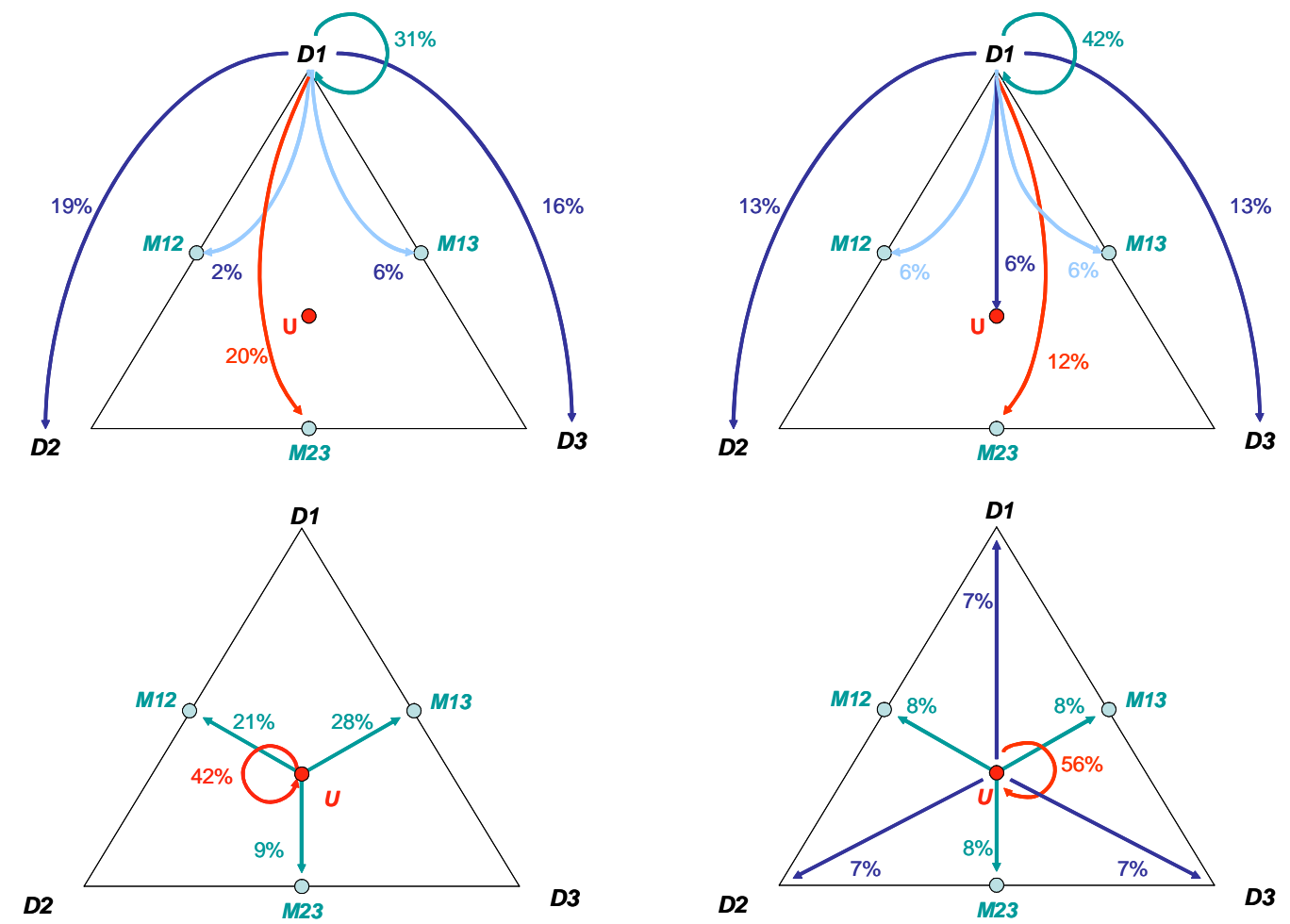

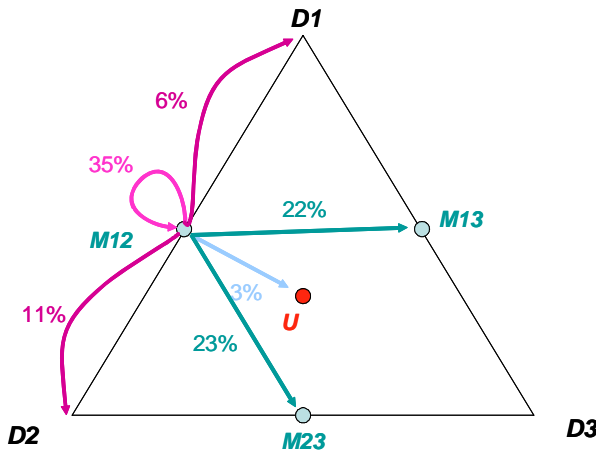

Empirical: $d=0.75$

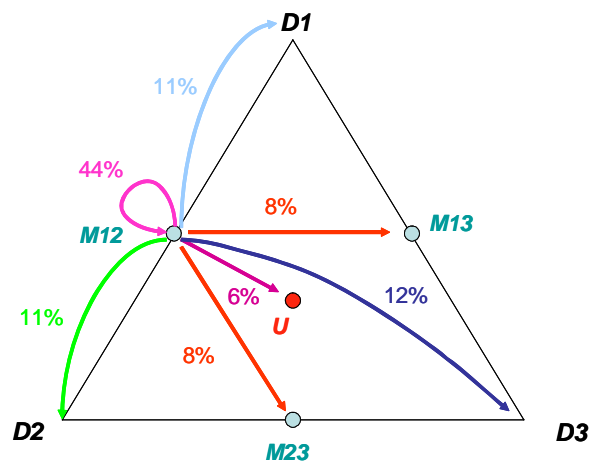

Weighted QRE: $d=0.75$

Figure 16: 
However, in the latter case, we actually observe zero transitions from U, which is not consistent with the theory.

From this estimation we conclude two main results. First, we can reject the hypothesis that the utility of the agents is linear with very high significance. In the distributive problem under analysis, concavity of the utility function seems to be an essential ingredient for an explanation that accounts for the empirical behavior that we observe in the lab. Second, the MLE model with concave preferences does a good job in accounting for the empirical evidence, as judged by comparing the observed transitions with the theoretical transitions at the estimated parameters of the MLE model.

\section{Concluding Remarks}

We studied dynamic committee behavior in multiperiod environments that are linked dynamically by the a sequence of status quo outcomes, where the status quo outcome in period $t$ is determined by the committee decision in period $t+1$. Such models capture the inertia of most naturally occurring, standing committees or legislatures. That is, outcomes in earlier periods have long run effects that are created simply by the powerful and special status quo position embodied in past decisions.

These environments were systematically varied in the laboratory, along three dimensions. First, we consider both continuous divide the dollar environments and highly constrained allocations. Second, for highly constrained settings, we consider Condorcet and non-Condorcet environments. Third, we look at the effect of committee "patience", or long run incentives, by varying the effective discount factor.

Many specific findings were reported in the data analysis. Most of these findings can be boiled down to six major results.

- Result 1: Outcomes have strong persistence, and this persistence tracks the theoretical prediction, but with some stochastic variation.

- Result 2: Voting behavior in all committees is overwhelmingly selfish and myopic.

- Result 3: Variations across treatments are due mainly to differences in proposal behavior.

These findings are in line with theoretical predictions, regardless of the specific assumptions of the model. Two results are at odd with the theory: 
- Result 4: Contrary to theoretical predictions, patient committees exhibit substantially different proposal behavior than impatient committees.

- Result 5: We observe more $\mathrm{U}$ and $\mathrm{M}$ outcomes than predicted by the Markov equilibrium with linear preferences.

The fact that in all treatments, we see more universal outcomes than expected based on the "standard" Markov equilibrium theory with linear preferences might tempt some to interpret as evidence of fairness and prosocial preferences. However, evidence from voting and proposal behavior does not seem to support such an interpretation. Voters consistently vote selfishly for outcomes that are extremely lopsided and unfair. Proposers tend to take advantage of their proposal power whenever the status quo allows them to do it.

We are able to show theoretically that universal outcomes will arise more often over time if preferences are concave. Using quantal response equilibrium we estimate the concavity of the utility function, and find it to be a significant factor. The coefficient of constant relative risk aversion is estimated on the pooled continuous data to be .5 , almost exactly the same as estimates of utility function curvature in other economics experiments. Based on a comparison of the empirical state transitions and the theoretical transition probabilities generated by the model estimates, we find:

- Result 6: The assumption of concave utility functions is highly significant statistically, and the concave utility model accounts reasonably well for the trajectory of allocations in our dynamic distributive politics environment.

The specific framework in this paper focused on dynamic distributive politics, but it is easily generalized to more complex political environments. In practice, in addition to purely distributive allocations, there are public good decisions, some of which have important dynamic components. Legislative committees make important decisions about the production and accumulation of real resources, for example public infrastructure and other durable public goods, and may finance these expenditures by running public debt, all of which has long run implications for future policy decisions. ${ }^{21}$ The next step of our research program is to extend the theoretical model in this direction and to collect and study experimental data in such environments.

\footnotetext{
${ }^{21}$ For a theoretical analysis of some of these issues, see Battaglini and Coate [2006], [2007a] and [2007b].
} 


\section{Appendix 1}

\section{Markov Quantal Response Equilibrium}

In this appendix, we define a Markov Quantal Response Equilibrium for the case of $J$ feasible alternatives (states) and $N$ legislators. We will focus on a particular version in which the quantal response function is logit, which we call Markov Logit Equilibrium $(M L E)$. As in the Markov Nash equilibrium, the state of the system is given by the status quo, and hence the state space is equal to the set of feasible alternatives. ${ }^{22}$

\section{VIII.0.1 Expected Utilities}

We define $v_{j i}$ to be the expected continuation utility of agent $i$ in state $j$ (so before the proposer is chosen), and a $J \times N$ matrix collecting all these values. All the following equations take $V$ as inputs.

We define $\sigma_{k}^{j i}(V)$ to be the probability of voting yes to a proposal $x_{k}$ in state $x_{j}$ by agent $i$. Let $P_{y}^{i}(V)$ and $F_{y}^{i}(V)$ be the probabilities that the proposal, respectively, passes or fail if $i$ votes yes in an equilibrium in which the value function is $V$. When, as in the experiment, $N=3$ we have $P_{y}^{i}(V)=1-\Pi_{l \neq i}(1-$

$\left.\sigma_{k}^{j l}(V)\right)$ and $F_{y}^{i}(V)=\Pi_{l \neq i}\left(1-\sigma_{k}^{j l}(V)\right)$. The expected utility from voting yes is $\left(x_{k i}+\delta v_{k i}\right) P_{y}^{i}(V)+$ $\left(x_{j i}+\delta v_{j i}\right) F_{y}^{i}(V)$. Similarly we can define the probabilities the proposal passes or fail if you vote no $P_{n}^{i}(V)$ and $P_{n}^{i}(V)$ that in the $\mathrm{N}=3$ case are $P_{n}^{i}(V)=\Pi_{l \neq i} \sigma_{k}^{j l}(V)$ and $F_{n}^{i}(V)=1-\Pi_{l \neq i} \sigma_{k}^{j l}(V)$. The expected utility from voting no is $\left(x_{k i}+\delta v_{k i}\right) P_{n}^{i}(V)+\left(x_{j i}+\delta v_{j i}\right) F_{n}^{i}(V)$. Since we are using Logit equilibrium, we use the logit quantal response function, which yields the following collection of MLE conditions characterizing the voting stage:

$$
\sigma_{k}^{j i}(V)=\frac{\exp \lambda\left\{\begin{array}{c}
\left(x_{k i}+\delta v_{k i}\right) P_{y}(V) \\
+\left(x_{j i}+\delta v_{j i}\right) F_{y}(V)
\end{array}\right\}}{\exp \lambda\left\{\begin{array}{c}
\left(x_{k i}+\delta v_{k i}\right) P_{y}(V) \\
+\left(x_{j i}+\delta v_{j i}\right) F_{y}(V)
\end{array}\right\}+\exp \lambda\left\{\begin{array}{c}
\left(x_{k i}+\delta v_{k i}\right) P_{n}(V) \\
+\left(x_{j i}+\delta v_{j i}\right) F_{n}(V)
\end{array}\right\}}
$$

where $\sigma_{k}^{j i}(V)$ is the probability that committee member $i$ would vote for proposal $x_{k}$ if the status quo is $x_{j}$.

\section{VIII.0.2 Proposal equilibrium conditions}

We define $u_{k}^{j i}(V)$ the expected utility of agent $i$ in state $x_{j}$ when $x_{k}$ is proposed (before the vote):

$$
\begin{aligned}
u_{k}^{j i}(V)= & \left(x_{k i}+\delta v_{k i}\right)\left[\sigma_{k}^{j i}(V) P_{y}^{i}(V)+\left(1-\sigma_{k}^{j i}(V)\right) P_{n}^{i}(V)\right] \\
& +\left(x_{j i}+\delta v_{j i}\right)\left[\sigma_{k}^{j i}(V) F_{y}^{i}(V)+\left(1-\sigma_{k}^{j i}(V)\right) F_{n}^{i}(V)\right] .
\end{aligned}
$$

\footnotetext{
${ }^{22}$ For an extensive discussion on the concept of Quantal Response Equilibrium in normal and extensive form games see McKelvey and Palfrey [1995] and [1998].
} 
Hence, the equilibrium conditions for the proposal stage are given by $\rho_{k}^{j i}(V)=\frac{\exp \left(-\lambda u_{k}^{j i}(V)\right)}{\sum_{k^{\prime}=1}^{J} \exp \left(-\lambda u_{k^{\prime}}^{j i}(V)\right)}$, where $\rho_{k}^{j i}(V)$ is the probability that committee member $i$ would propose $x_{k}$ in state $x_{j}$.

\section{VIII.0.3 The fixed point problem}

The following expression defines a $N \times J$ equations that map expected utilities $V$ to expected utilities $V$.

$$
v_{j i}=\sum_{l=1}^{N} \alpha_{i}\left[\sum_{k=1}^{J} \rho^{j k}(V) u_{k}^{j i}(V)\right]
$$

where $\alpha_{i}$ is the probability that agent $i$ is selected as proposer. The fixed points of this equation are MLE of the bargaining game. The fixed-point of (7) at a given $\lambda^{\circ}$ is computed by homotopy methods. We know the fixpoint of (7) at $\lambda=0$. Though (7) is not a contraction, it behaves as a contraction in a neighborhood of a fixed-point. We can therefore find the fixed-point at $\lambda^{\circ}$ by tracing the fixed-points of (7) as $\lambda$ gradually increases up to $\lambda^{\circ}$. In the analysis of an approximate Nash equilibrium in Section III we assume $\lambda^{\circ}=20$.

\section{VIII.1 Proof of Proposition 1}

We first show that the strategies described in the proposition are an equilibrium for any increasing utility $U$. We can normalize utility by $U(0)=0, U(30)=1$, and $U(20)=a \in(0,1)$. Then the value functions for player $i$ can be written:

$$
u_{i}\left(x_{0}\right)=a+\delta v_{i}\left(x_{0}\right), \quad u_{i}\left(x_{i}\right)=\delta v_{i}\left(x_{i}\right), \quad u_{i}\left(x_{-i}\right)=1+\delta v_{i}\left(x_{-i}\right),
$$

where $v_{i}(x)$ is the continuation expected utility of being in state $x$. The continuation values are determined by the recognition probabilities $(1 / 3$ each) and the proposal strategies, and the voting strategies. By hypothesis, these strategies are as stated in Proposition 1, and therefore we get

$$
v_{i}\left(x_{0}\right)=\frac{1}{3} u_{i}\left(x_{i}\right)+\frac{2}{3} u_{i}\left(x_{-i}\right), \quad v_{i}\left(x_{i}\right)=\frac{2}{3} u_{i}\left(x_{i}\right)+\frac{1}{3} u_{i}\left(x_{-i}\right), \quad v_{i}\left(x_{-i}\right)=\frac{1}{6} u_{i}\left(x_{i}\right)+\frac{5}{6} u_{i}\left(x_{-i}\right) .
$$

From here, it is straightforward to show that, for all $a \in(0,1)$, the value functions are ordered

$$
u_{i}\left(x_{-i}\right)>u_{i}\left(x_{0}\right)>u_{i}\left(x_{i}\right),
$$

which is sufficient to show that the strategies described in Proposition 1 form an equilibrium. There are two steps. first, we show that $u_{i}\left(x_{-i}\right)>u_{i}\left(x_{i}\right)$. To see this, subtract the second equation in 8 from the 
third equation, to get

$$
u_{i}\left(x_{-i}\right)-u_{i}\left(x_{i}\right)=1+\delta\left[v_{i}\left(x_{-i}\right)-v_{i}\left(x_{i}\right)\right]=1+\frac{1}{2} \delta\left[u_{i}\left(x_{-i}\right)-u_{i}\left(x_{i}\right)\right]
$$

with the second step resulting from the substitution of the last two equations of 9 . Hence: $u_{i}\left(x_{-i}\right)-$ $u_{i}\left(x_{i}\right)=\frac{2}{2-\delta}>0$. To see that $u_{i}\left(x_{-i}\right)-u_{i}\left(x_{0}\right)>0$, observe that from 8 we get:

$$
u_{i}\left(x_{-i}\right)-u_{i}\left(x_{0}\right)=(1-a)+\delta\left[v_{i}\left(x_{-i}\right)-v_{i}\left(x_{0}\right)\right]
$$

Using 9, the result follows immediately. It is also easy to show, using exactly the same line of argument, that the same result (rotating minimum winning coalitions) extends to any odd number $N$ of committee members, where the set of alternatives includes all equal-split minimum winning coalitions, plus the universal outcome.

Note that in any equilibrium in which (10) is true, strategies must be as described in the proposition. So to establish uniqueness when utilities are linear, we only need to show that (10) must be satisfied in an equilibrium (as defined in Section II). It is easy to see that in any (symmetric) equilibrium the utility of each agent is identical in state $x_{0}: u_{i}\left(x_{0}\right)=u_{j}\left(x_{0}\right) \forall i, j$. Moreover, by symmetry, we also have that $u_{i}\left(x_{j}\right)=u_{k}\left(x_{j}\right)$ for $i, k \neq j$, and $u_{i}\left(x_{i}\right)=u_{j}\left(x_{j}\right)$ for $\forall j, i$. Finally note that, with linear utilities, for any state $x$ we must have $\sum_{i=1}^{3} u_{i}(x)=\frac{60}{1-\delta}$ for any $x$. Together with symmetry, these conditions imply that $u_{i}\left(x_{0}\right)=\frac{20}{1-\delta} \forall i$.

Assume first that in some equilibrium $u_{i}\left(x_{j}\right)<u_{i}\left(x_{0}\right)$ for $j \neq i$. In this case it must be that $u_{i}\left(x_{i}\right)<u_{i}\left(x_{j}\right)$. Assume not. Then we would have that the worst continuation utility for agent $i$ in state $x_{j}$ is $u_{i}\left(x_{j}\right)$. This would imply $u_{i}\left(x_{j}\right) \geq 30+\delta u_{i}\left(x_{j}\right)$, i.e. $u_{i}\left(x_{j}\right) \geq \frac{30}{1-\delta}>\frac{20}{1-\delta}=u_{i}\left(x_{0}\right)$, a contradiction. If $u_{i}\left(x_{i}\right)<u_{i}\left(x_{j}\right)$ then we have that

$$
60=(1-\delta) \sum_{i=1}^{3} u_{i}\left(x_{j}\right)=(1-\delta)\left[2 u_{i}\left(x_{j}\right)+u_{j}\left(x_{j}\right)\right]<3(1-\delta) u_{i}\left(x_{j}\right)
$$

since $u_{j}\left(x_{j}\right)=u_{i}\left(x_{i}\right)$. This implies that $u_{i}\left(x_{j}\right)>u_{i}\left(x_{0}\right)$, a contradiction. We conclude that $u_{i}\left(x_{j}\right) \geq$ $u_{i}\left(x_{0}\right)$.

We now show that $u_{i}\left(x_{j}\right)>u_{i}\left(x_{0}\right)$. Assume not, so that $u_{i}\left(x_{j}\right)=u_{i}\left(x_{0}\right)$. Assume first that $u_{i}\left(x_{i}\right) \geq u_{i}\left(x_{0}\right)$. In this case, the continuation utility for agent agent $i$ in state $x_{j}$ is never below $u_{i}\left(x_{0}\right)$, so $u_{i}\left(x_{j}\right) \geq 30+\delta u_{i}\left(x_{0}\right)=10+u_{i}\left(x_{0}\right)>u_{i}\left(x_{0}\right)$, a contradiction. Assume now that $u_{i}\left(x_{i}\right)<u_{i}\left(x_{0}\right)$. 
Then, for $j \neq 0$, we have

$$
\begin{aligned}
60 & =(1-\delta) \sum_{i=1}^{3} u_{i}\left(x_{j}\right)=(1-\delta)\left[2 u_{i}\left(x_{j}\right)+u_{j}\left(x_{j}\right)\right] \\
& <(1-\delta)\left[2 u_{i}\left(x_{j}\right)+u_{j}\left(x_{0}\right)\right]=3(1-\delta) u_{i}\left(x_{0}\right)
\end{aligned}
$$

which implies that $u_{i}\left(x_{0}\right)>\frac{20}{1-\delta}$, a contradiction.

Finally, assume that $u_{i}\left(x_{i}\right) \geq u_{i}\left(x_{0}\right)$. Then, using the same logic as before, we have that for $j \neq 0$ :

$60=(1-\delta)\left[2 u_{i}\left(x_{j}\right)+u_{j}\left(x_{j}\right)\right]>(1-\delta) \sum_{i=1}^{3} u_{i}\left(x_{0}\right)$, a contradiction. We conclude that $u_{i}\left(x_{j}\right)>u_{i}\left(x_{0}\right)>$ $u_{i}\left(x_{i}\right)$, which proves the result.

\section{VIII.2 Proof of Proposition 2}

If strategies are myopic as defined in Section III.2.2 the value function is described by (3), (4) and (5). It is easy to verify that $v_{i}\left(x_{i}\right)>v_{i}\left(x_{0}\right)>v_{i}\left(x_{j}\right)$. This implies that the voting behavior described in a myopic strategy profile is optimal. Given this, the weakly stage undominated strategy for an agent $i$ is always to propose $x_{i}$ in state $x_{i}$ or $x_{0}$. Consider now the case of an agent $i$ in state $x_{j}$. It is easy to verify that it is never optimal to propose $x_{k}$ for $k \neq i, 0$ : this choice would certainly yield a lower utility than proposing, for example, $x_{0}$, which would always win. When the state is $x_{j}$, it is optimal for agent $i$ to propose $x_{i}$ if it yields higher expected utility than $x_{0}$, that is if: $\left(\frac{45}{2}+\frac{\delta}{2}\left(v_{i}\left(x_{i}\right)+v_{i}\left(x_{j}\right)\right)\right) \geq \frac{20}{(1-\delta)}$. The right hand side of this inequality is the utility if $x_{0}$ is proposed (given that it wins with probability one), and the left hand side is the expected utility of proposing $x_{i}$, which wins only with probability $\frac{1}{2}$. Given (4), (5), it can be verified that this inequality is true for all $\delta \in[0,1]$. We conclude that the myopic strategies described in Section 3.2.2 are an equilibrium.

We now prove that they must be the unique equilibrium. Proceeding as in Proposition 1, we can show that a symmetric equilibrium must be monotonic: so $u_{i}\left(x_{i}\right)>u_{i}\left(x_{0}\right)>u_{i}\left(x_{j}\right)$. We therefore only need to prove that any monotonic equilibrium must adopt myopic strategies as defined in Section III.2.2. It is easy to see that any monotonic equilibrium must have the same voting behavior as described in the myopic strategies. Given this it is also immediate to see that proposal behavior must be as described in myopic strategies for each agent $i$ in states $x_{i}$ and $x_{0}$. Consider now proposal behavior of agent $i$ in state $x_{j}$. As before we can rule out the case in which $x_{k}$ for $k \neq i, 0$ is proposed. Assume that the agent proposes $x_{0}$ with positive probability, say with probability $a$. In this case it must be that:

$$
\left(\frac{45}{2}+\frac{\delta}{2}\left(v_{i}\left(x_{i}\right)+v_{i}\left(x_{j}\right)\right)\right) \leq \frac{20}{(1-\delta)}
$$


Moreover it must be that in any state the sum of payoffs sums up to $\frac{60}{(1-\delta)}$, so by symmetry:

$$
v_{i}\left(x_{i}\right)+2 v_{i}\left(x_{j}\right)=\frac{60}{(1-\delta)} .
$$

From (11)-(12), we obtain: $v_{i}\left(x_{j}\right) \geq \frac{15 \delta+5}{\delta(1-\delta)}$. Using this fact, we note that: $u_{i}\left(x_{j}\right)=15+\delta v_{i}\left(x_{j}\right) \geq$ $\frac{20}{(1-\delta)}=u_{i}\left(x_{0}\right)$, a contradiction. We conclude that a symmetric equilibrium must be in myopic strategies.

\section{References}

[1] Azzimonti, M. (2006), "On the Dynamic Inefficiency of Governments," Mimeo.

[2] Baron, David P. (1996) "A Dynamic Theory of Collective Goods Procedures," American Political Science Review, 90(June):316-30.

[3] Baron, David P. and John A. Ferejohn (1989) "Bargaining in Legislatures," American Political Science Review, 83(June):1181-1206.

[4] Baron, David P. and Michael Herron (2003) "A Dynamic Model of Multidimensional Collective Choice." in Computational Models of Political Economy, K. Kollman, J. Miller and S. Page eds. MIT Press: Cambridge: 13-47.

[5] Battaglini, Marco and Stephen Coate (2006) "Inefficiency in Legislative Policymaking: A Dynamic Analysis," American Economic Review, 97(March):118-49.

[6] Battaglini, Marco and Stephen Coate (2007a) "A Dynamic Theory of Public Spending, Taxation and Debt," American Economic Review, forthcoming.

[7] Battaglini, Marco and Stephen Coate (2007b) "Fiscal Policy over the Real Business Cycle: A Positive Theory," mimeo.

[8] Campo, Sandra., Emmaneul Guerre, Isabelle Perrigne, and Quang Vuong (2000) "Semiparametric Estimation of First-Price Auctions with Risk Averse Bidders," Working Paper, Penn State University.

[9] Diermeier, D. and P. Fong (2007), "Dynamic Legislative Bargaining with Reconsideration," mimeo.

[10] Diermeier, Daniel and Rebecca Morton (2006) "Experiments in Majoritarian Bargaining," in Social Choice and Strategic Decisions: Essays in Honor of Jeffrey S. Banks, D. Austen-Smith and J. Duggan eds. Springer: Heidelberg: 201-26. 
[11] Diermeier, Daniel and Sean Gailmard (2006) "Self-Interest, Inequality, and Entitlement in Majoritarian Decision-Making," Quarterly Journal of Political Science, 1: 327-350.

[12] Duggan, Johm and Tasos Kalandrakis (2007) "A Dynamic Model of Legislative Bargaining," mimeo.

[13] Frechette, Guillaume, John H. Kagel and Steven F. Lehrer (2003) "Bargaining in Legislatures: An Experimental Investigation of Open versus Closed Amendment Rules" , American Political Science Review, 97(May): 221-32.

[14] Frechette, Guillaume, John H. Kagel and Massimo Morelli (2005a) "Gamson's Law versus NonCooperative Bargaining Theory", Games and Economic Behavior, 51(May): 365-90.

[15] Frechette, Guillaume, John H. Kagel and Massimo Morelli (2005b) "Nominal Bargaining Power, Selection Protocol, and Discounting in Legislative Bargaining", Journal of Public Economics, 89(August): 1497-1517.

[16] Frechette, Guillaume, John H. Kagel and Massimo Morelli (2005c) "Behavioral Identification in Coalitional Bargaining: An Experimental Analysis of Demand Bargaining and Alternating Offers", Econometrica, 73 (October): 1893-1938.

[17] Frechette, Guillaume, John H. Kagel and Massimo Morelli (2007) "Pork Versus Public Goods: An Experimental Study of Public Good Provision Within a Legislative Bargaining Framework." mimeo.

[18] Goeree, Jacob K., Thomas R. Palfrey, and Charles A. Holt (2002). "Quantal Response Equilibrium and Overbidding in First Price Auctions," Journal of Economic Theory 104(1): 247-72.

[19] Goeree, Jacob K., Thomas R. Palfrey, and Charles A. Holt (2003). "Risk Averse Behavior in Generalized Matching Pennies Games" Games and Economic Behavior 45(1): 97-113.

[20] Holt, Charles A. and Susan Laury (2002). "Risk Aversion and Incentive Effects." American Economic Review 92(5): 1644-1655.

[21] Kalandrakis, Tasos (2004). "A Three Player Dynamic Majoritarian Bargaining Game", Journal of Economic Theory, 16(2): 294-322.

[22] Kalandrakis, Tasos (2005). "Majority Rule Dynamics with Endogenous Status Quo", Working Paper, University of Rochester, http://mail.rochester.edu/〜akalandr/download/mrbg.pdf. 
[23] McKelvey, Richard D. (1991). "An Experimental Test of a Stochastic Game Model of Committee Bargaining," in Laboratory Research in Political Economy, T. Palfrey ed. University of Michigan Press: Ann Arbor: 139-69.

[24] McKelvey, Richard D. and Thomas R. Palfrey (1995) "Quantal Response Equilibria for Normal Form Games," Games and Economic Behavior, 10(July):6-38.

[25] McKelvey, Richard D. and Thomas R. Palfrey (1998) "Quantal Response Equilibria for Extensive Form Games," Experimental Economics, 1, 9-41.

[26] Penn, Elizabeth M. (2007) "A Model of Farsighted Voting" Working Paper, Harvard University, http://www.people.fas.harvard.edu/ epenn/remarkov.pdf. 